\title{
Reciprocal processes. A measure-theoretical point of view*
}

\author{
Christian Léonard ${ }^{\dagger}$ \\ Modal-X. Université Paris Ouest. Bât. G, 200 av. de la République \\ 92001 Nanterre, France \\ e-mail: christian.leonard@u-paris10.fr \\ Sylvie Rœlly \\ Institut für Mathematik der Universität Potsdam. Am Neuen Palais 10 \\ 14469 Potsdam, Germany \\ e-mail: roelly@math.uni-potsdam.de \\ and \\ Jean-Claude Zambrini ${ }^{\ddagger}$ \\ GFM, Universidade de Lisboa, Av. Prof. Gama Pinto 2 \\ 1649-003 Lisboa, Portugal \\ e-mail: zambrini@cii.fc.ul.pt
}

\begin{abstract}
The bridges of a Markov process are also Markov. But an arbitrary mixture of these bridges fails to be Markov in general. However, it still enjoys the interesting properties of a reciprocal process.

The structures of Markov and reciprocal processes are recalled with emphasis on their time-symmetries. A review of the main properties of the reciprocal processes is presented. Our measure-theoretical approach allows for a unified treatment of the diffusion and jump processes. Abstract results are illustrated by several examples and counter-examples.
\end{abstract}

Keywords and phrases: Markov process, reciprocal process, Markov bridge, time-symmetry, entropy minimization.

Received August 2013.

\section{Contents}

Introduction . . . . . . . . . . . . . . . . . . 238

1 Time-symmetry of Markov measures . . . . . . . . . . . . . . . 240

1.1 Definition and basic properties ... . . . . . . . . 240

1.2 Path measures dominated by a Markov measure . . . . . . . . 243

1.3 A fundamental example: Bridges of a Markov measure . . . . . . 245

*The authors are thankful to the UFA-DFH for its support through the French-German Doktorandenkolleg CDFA 01-06. They also thank the anonymous reviewer for it valuable input on the article.

${ }^{\dagger}$ Partly supported by the ANR projects GeMeCoD (ANR 2011 BS01 007 01) and STAB.

‡Partly supported by the project PTDC/MAT/120354/2010. 
2 Reciprocal measures . . . . . . . . . . . . . . . . . 250

2.1 Definition and basic properties . . . . . . . . . . . . 251

2.2 Pinning leads back to the Markov property . . . . . . . . . . . . 254

2.3 Mixing properly preserves the reciprocal property . . . . . . . . . 254

2.4 Reciprocal class associated with a measure . . . . . . . . . . 256

2.5 Time reversal and reciprocal classes . . . . . . . . . . . 257

2.6 Reciprocal subclass of dominated measures . . . . . . . . . 258

2.7 Markov measures of a reciprocal class . . . . . . . . . . . . 259

3 Reciprocal measures are solutions of entropy minimizing problems . . 261

3.1 Schrödinger's problem . . . . . . . . . . . . . . . . . 262

3.2 A modification of Schrödinger's problem . . . . . . . . . . . 265

Acknowledgements . . . . . . . . . . . . . . . . . 266

References . . . . . . . . . . . . . . . . . . . 267

\section{Introduction}

The Markov property is a standard probabilistic notion since its formalization at the beginning of the 20th century. It was presented for the first time in a time-symmetric way by Doob [19] in 1953, see (1.1). This remarkable point of view, which is often replaced by its time-asymmetric counterpart, was developed further by numerous authors, see the references in the monograph by Chung and Walsh [9] and also Wentzell [53].

Two decades after Markov, Bernstein [3] introduced in the particular framework of diffusion processes the more general notion of reciprocal process ${ }^{1}$ which is also time-symmetric, by its very definition. During his talk at the International Congress in Zürich, he qualified the dynamics of reciprocal processes as stochastiquement parfaite ${ }^{2}$. Bernstein's motivation for introducing these timesymmetric notion of random process is rooted into an outstandingly insightful article by Schrödinger [45] entitled "Über die Umkehrung der Naturgesetze" published in 1931, allowing a clear analogy with quantum dynamics, see Section 3.

The particular case of Gaussian reciprocal processes was extensively treated by Jamison [26]. This was also undertaken by Chay [7] who named these processes "quasi-Markov random fields" since they are random fields defined on a compact time interval. See further comments and references at the beginning of Section 2 and Subsection 2.4. Later, in the excellent seminal paper [27] entitled "Reciprocal processes", Jamison provided a rigorous definition of these processes and derived their main properties in an abstract setting. This led to many studies in specific contexts, such as diffusion processes [49, 50, 16, 48], Lévy processes [40] or pure jump processes [14, 13].

This review paper revisits [27] and provides some new results. We present a unifying measure-theoretical approach to reciprocal processes. Unlike Jamison, who worked with finite-dimensional distributions using the concept of reciprocal

\footnotetext{
${ }^{1}$ This terminology is due to Bernstein. They are also sometimes called Bernstein processes. ${ }^{2}$ Stochastically perfect.
} 
transition probability function, we look at Markov and reciprocal processes as path measures, i.e. measures on the path space.

Theorem 2.14 illustrates our perspective. Its statement gives a characterization of the reciprocal measures which are dominated by a given Markov measure in terms of time-symmetric versions of Doob's $h$-transforms. We also illustrate our abstract results with several examples and counter-examples.

A possible extension

We focus onto probability path measures. As a consequence, in the present paper we drop the word probability: any path probability measure, Markov probability measure or reciprocal probability measure is simply called a path measure, a Markov measure or a reciprocal measure.

Our results can easily be extended to $\sigma$-finite path measures, e.g. processes admitting an unbounded measure as their initial law. For further detail about this generalized framework, see [33].

\section{Outline of the paper}

Section 1 is devoted to the structure of Markov measures and to their timesymmetries. Reciprocal measures are introduced at Section 2 and their relationship with Markov measures is investigated. At Section 3, we sketch the tight connection between reciprocal classes and some specific entropy minimization problems. So doing, we step back to Schrödinger's way of looking at some statistical physics problems with a time-symmetric viewpoint.

\section{Notation}

We consider the set $\Omega=D([0,1], \mathcal{X}) \subset \mathcal{X}^{[0,1]}$ of càdlàg paths defined on the finite time interval $[0,1]$ with state space $\mathcal{X}$, which is assumed to be Polish and equipped with its Borel $\sigma$-algebra. As usual $\Omega$ is endowed with the canonical filtration $\mathcal{A}$ generated by the canonical process $X=\left(X_{t}\right)_{t \in[0,1]}$ :

$$
X_{t}(\omega):=\omega_{t}, \quad \omega=\left(\omega_{s}\right)_{s \in[0,1]} \in \Omega, t \in[0,1] .
$$

For any subset $\mathcal{S} \subset[0,1]$ and for any measure $P$ on $\Omega$ one denotes

- $X_{\mathcal{S}}=\left(X_{s}\right)_{s \in \mathcal{S}}$ the canonical process restricted to $\mathcal{S}$,

- $\mathcal{A}_{\mathcal{S}}=\sigma\left(X_{s} ; s \in \mathcal{S}\right)$ the $\sigma$-algebra of the events observed during $\mathcal{S}$,

- $P_{\mathcal{S}}=\left(X_{\mathcal{S}}\right)_{\#} P$ the restriction of $P$ to $\Omega_{\mathcal{S}}:=X_{\mathcal{S}}(\Omega)$.

We have denoted $f_{\#} m:=m \circ f^{-1}$ the image of the measure $m$ with respect to the measurable mapping $f$.

For $\mathcal{S}=[s, u] \subset[0,1]$ we use the particular notations:

- $X_{[s, u]}:=\left(X_{t} ; s \leq t \leq u\right)$

- $\mathcal{A}_{[s, u]}:=\sigma\left(X_{[s, u]}\right)$, the $\sigma$-algebra generated by the events that occurred between time $s$ and time $u$ 
- $\mathcal{A}_{s}:=\sigma\left(X_{s}\right)$, the $\sigma$-algebra generated by the events that occur at time $s$

- $P_{s}:=\left(X_{s}\right)_{\#} P$ is the projection of $P$ at time $s$

- $P_{s u}:=\left(X_{s}, X_{u}\right)_{\#} P$ is the marginal law of $P$ at times $s$ and $u$ simultaneously ( $P_{01}$ is therefore the endpoint marginal law of the process)

- $P_{[s, u]}:=\left(X_{[s, u]}\right)_{\#} P$ is the projection of $P$ on the time interval $[s, u]$

The measure

$$
P^{*}=\left(X^{*}\right)_{\#} P
$$

is the law under $P$ of the càdlàg transform $X^{*}: \Omega \rightarrow \Omega$ of the time reversed canonical process defined by $X_{t}^{*}:=\lim _{h \rightarrow 0, h \geq 0} X_{(1-t+h)}$ for any $0<t<1$. To avoid technical troubles with the endpoint positions, as far as $X^{*}$ is at stake, it is assumed for simplicity that

$$
P\left(X_{0}=X_{0^{+}}\right)=P\left(X_{1}=X_{1^{-}}\right)=1 .
$$

\section{Time-symmetry of Markov measures}

We present structural properties of both Markov probability measures and their bridges. Emphasis is put on their time-symmetry which has already been studied in specific frameworks, see for instance [9].

\subsection{Definition and basic properties}

Let us begin with the symmetric definition of the Markov property.

Definition 1.1 (Markov measure). A probability measure $P$ on $\Omega$ is said to be Markov if for any $t \in[0,1]$ and for any events $A \in \mathcal{A}_{[0, t]}, B \in \mathcal{A}_{[t, 1]}$

$$
P\left(A \cap B \mid X_{t}\right)=P\left(A \mid X_{t}\right) P\left(B \mid X_{t}\right), \quad P \text {-a.e. }
$$

This means that, knowing the present state $X_{t}$, the future and past informations $\mathcal{A}_{[t, 1]}$ and $\mathcal{A}_{[0, t]}$, are $P$-independent.

In Theorem 1.2 below, we recall equivalent descriptions of the Markov property. In particular, the standard identity (2) states that a Markov process forgets its past history.

Theorem 1.2. Let $P$ be a probability measure on $\Omega$. Then the following are equivalent:

(1) The measure $P$ is Markov.

$\left(1^{*}\right)$ The time-reversed measure $P^{*}$ is Markov (assuming (0.1)).

(2) For all $0 \leq t \leq 1$ and all sets $B \in \mathcal{A}_{[t, 1]}$,

$$
P\left(B \mid X_{[0, t]}\right)=P\left(B \mid X_{t}\right), \quad P \text {-a.e. }
$$

(2*) For all $0 \leq t \leq 1$ and all sets $A \in \mathcal{A}_{[0, t]}$,

$$
P\left(A \mid X_{[t, 1]}\right)=P\left(A \mid X_{t}\right), \quad P \text {-a.e. }
$$


(3) For all $0 \leq s \leq u \leq 1$ and all sets $A \in \mathcal{A}_{[0, s]}, C \in \mathcal{A}_{[u, 1]}$

$$
P\left(A \cap C \mid X_{[s, u]}\right)=P\left(A \mid X_{s}\right) P\left(C \mid X_{u}\right), \quad P \text {-a.e. }
$$

Proof. Let us prove $(3) \Rightarrow(1) \Rightarrow(2) \Rightarrow(3)$.

- Proof of $(3) \Rightarrow(1)$. It is clear by taking $s=u$.

- Proof of $(2) \Rightarrow(3)$. For all sets $A \in \mathcal{A}_{[0, s]}$ and $C \in \mathcal{A}_{[u, 1]}$ and all sets $B \in \mathcal{A}_{[s, u]}$, the equality

$$
P(A \cap B \cap C)=E\left[\mathbf{1}_{B} P\left(A \cap C \mid X_{[s, u]}\right)\right]
$$

holds. On the other hand,

$$
\begin{aligned}
P(A \cap B \cap C) & =E\left[P\left(A \cap B \cap C \mid X_{[0, u]}\right)\right] \\
& =E\left[\mathbf{1}_{A} \mathbf{1}_{B} P\left(C \mid X_{[0, u]}\right)\right] \\
& =E\left[\mathbf{1}_{A} \mathbf{1}_{B} P\left(C \mid X_{[s, u]}\right)\right] \\
& =E\left[\mathbf{1}_{B} P\left(A \mid X_{[s, u]}\right) P\left(C \mid X_{[s, u]}\right)\right]
\end{aligned}
$$

where property (2) is used in the third equality. Therefore

$$
P\left(A \cap C \mid X_{[s, u]}\right)=P\left(A \mid X_{[s, u]}\right) P\left(C \mid X_{[s, u]}\right) .
$$

- Proof of $(1) \Rightarrow(2)$. It is based on standard properties of conditional independence. Nevertheless, for the sake of completeness, we sketch it. Let us show that if (1.1) is satisfied then $P$ forgets its past history. Let $A \in \mathcal{A}_{[0, t]}$ and $B \in \mathcal{A}_{[t, 1]}$ be some events.

$$
E\left[\mathbf{1}_{A} P\left(B \mid X_{[0, t]}\right)\right]=P(A \cap B)=E\left(P\left(A \cap B \mid X_{t}\right)\right)=E\left[P\left(A \mid X_{t}\right) P\left(B \mid X_{t}\right)\right] .
$$

On the other hand,

$$
E\left[\mathbf{1}_{A} P\left(B \mid X_{t}\right)\right]=E\left[P\left(A \mid X_{t}\right) P\left(B \mid X_{t}\right)\right] .
$$

One obtains for any set $A \in \mathcal{A}_{[0, t]}, E\left[\mathbf{1}_{A} P\left(B \mid X_{[0, t]}\right)\right]=E\left[\mathbf{1}_{A} P\left(B \mid X_{t}\right)\right]$, which implies that $P\left(B \mid X_{[0, t]}\right)=P\left(B \mid X_{t}\right)$. This completes the proof of $(1) \Rightarrow(2)$, together with the proof of $(1) \Leftrightarrow(2) \Leftrightarrow(3)$.

Eventually the symmetry of the formulation of (3) leads to the equivalence between $(2)$ and $\left(1^{*}\right)$. Assertion $\left(2^{*}\right)$ is equivalent to $(2)$ by usual symmetry of conditional independence.

The first proof of $(1) \Leftrightarrow(2)$ appears in the monograph by Doob [19, Eqs (6.8) \& (6.8')]. Then, Dynkin [21] and Chung [8, Thm. 9.2.4] took it over. Meyer already remarked in [35] that the Markov property is invariant under time reversal. Let us cite him: Soit $\left(X_{t}\right)$ un processus de Markov; les tribus $\sigma\left(X_{[t, 1]}\right)$ et $\sigma\left(X_{[0, t]}\right)$ jouant des rôles symétriques dans la définition de l'indépendance conditionnelle (1.1), le processus $\left(X_{t}\right)$ reste markovien lorsqu'on "renverse le sens du temps". 
The proof of $(1) \Leftrightarrow\left(1^{*}\right)$ does not require any assumption about the regularity of the sample paths. It is not important that $\Omega$ is a set of càdlàg paths. Therefore, if $\Omega$ is replaced by the set $\mathcal{X}^{[0,1]}$ of all paths, this identity holds with $P^{*}=$ $\left(X^{*}\right)_{\#} P$ and $X_{t}^{*}:=X_{1-t}, 0 \leq t \leq 1$, without assuming $(0.1)$.

Identity (2), called left-sided Markov property, is often used as the definition of the Markov property. It may create the inaccurate delusion (frequent in the context of statistical physics) that the Markov property is time-asymmetric.

Since each Markov process can be defined via its forward and backward transition probability kernels, we recall how to construct them in a symmetric way.

Definitions 1.3. Let $P$ be a Markov measure.

1. The forward transition probability kernel associated with $P$ is the family of conditional measures $(p(s, x ; t, \cdot) ; 0 \leq s \leq t \leq 1, x \in \mathcal{X})$ defined for any $0 \leq s \leq t \leq 1$, and $P_{s}$-almost all $x$, by

$$
p(s, x ; t, d y)=P\left(X_{t} \in d y \mid X_{s}=x\right)
$$

2. The backward transition probability kernel associated with $P$ is the family of conditional measures $\left(p^{*}(s, \cdot ; t, y) ; 0 \leq s \leq t \leq 1, y \in \mathcal{X}\right)$ defined for any $0 \leq s \leq t \leq 1$, and $P_{t}$-almost all $y$, by

$$
p^{*}(s, d x ; t, y):=P\left(X_{s} \in d x \mid X_{t}=y\right)
$$

Since these kernels satisfy the celebrated Chapman-Kolmogorov relations

$$
\begin{aligned}
\forall 0 \leq s \leq t \leq u \leq 1 & \\
p(s, x ; u, \cdot) & =\int_{\mathcal{X}} p(s, x ; t, d y) p(t, y ; u, \cdot) \text { for } P_{s^{-a . a . ~} x} \\
p^{*}(s, \cdot ; u, z) & =\int_{\mathcal{X}} p^{*}(s, \cdot ; t, y) p^{*}(t, d y ; u, z) \text { for } P_{u} \text {-a.a. } z
\end{aligned}
$$

one can construct the measure $P$ in the following way.

Proposition 1.4. The Markov measure $P$ is uniquely determined by one time marginal $P_{u}$ at some time $u \in[0,1]$, its forward transition probability kernels starting from time $u,(p(s, x ; t, \cdot) ; u \leq s \leq t \leq 1, x \in \mathcal{X})$ and the backward transition probability kernels since time $u,\left(p^{*}(s, \cdot ; t, y) ; 0 \leq s \leq t \leq u, y \in \mathcal{X}\right)$.

Indeed, for any $0 \leq s_{1} \leq \cdots \leq s_{k} \leq u \leq t_{1} \leq \cdots \leq t_{l} \leq 1$ and $k, l \geq 1$, the finite dimensional projection of $P$ are given by

$$
P_{s_{1}, \ldots, s_{k}, u, t_{1}, \ldots, t_{l}}=p_{s_{1} ; s_{2}}^{*} \otimes \cdots \otimes p_{s_{k} ; u}^{*} \otimes P_{u} \otimes p_{u ; t_{1}} \otimes \cdots \otimes p_{t_{l-1} ; t_{l}}
$$

where we used the following intuitive notation

$$
P_{u} \otimes p_{u ; t}(d x, d y):=P_{u}(d x) p(u, x ; t, d y)
$$




\subsection{Path measures dominated by a Markov measure}

We consider the problem of knowing whether a path measure which is dominated by a reference Markov measure inherits its Markov property. The following result states a criterion in terms of the multiplicative structure of the Radon-Nikodym derivative on the path space. This question was posed in the general background of continuous time Markov fields in the 1970' and solved by Dang Ngoc and Yor in [18, Prop. 4.3(b)]. Some abstract variant of this result also appeared in [51, Thm. 3.6]. In the simpler framework of processes indexed by a discrete time, we refer the reader to Georgii's extended monograph [25, Ch. 10, 11].

Theorem 1.5. Let $R$ be a reference Markov measure and let $P \ll R$, a probability measure dominated by $R$. Then the following statements are equivalent.

(1) The measure $P$ is Markov.

(2) For any time $t \in[0,1]$, the Radon-Nikodym derivative of $P$ with respect to $R$ factorizes in the following way:

$$
\frac{d P}{d R}=\alpha_{t} \beta_{t}, \quad R \text {-a.e. }
$$

where $\alpha_{t}$ and $\beta_{t}$ are respectively nonnegative $\mathcal{A}_{[0, t]}{ }^{-m e a s u r a b l e}$ and $\mathcal{A}_{[t, 1]^{-}}$ measurable functions.

Proof. For an alternate proof, see [18, Prop. 4.3].

- Proof of $(2) \Rightarrow(1)$. Take two events, $A \in \mathcal{A}_{[0, t]}$ and $B \in \mathcal{A}_{[t, 1]}$. In terms of Definition 1.1, we have to show that

$$
P\left(A \cap B \mid X_{t}\right)=P\left(A \mid X_{t}\right) P\left(B \mid X_{t}\right), \quad P \text {-a.e. }
$$

To this aim, note that although the product $\alpha_{t} \beta_{t}$ is $R$-integrable, it is not clear why $\alpha_{t}$ or $\beta_{t}$ should be separately integrable. To prove this required integrability, one may use the following lemma of integration theory which assures the $R\left(\cdot \mid X_{t}\right)$-integrability of the functions $\alpha_{t}$ and $\beta_{t}, P$-a.e.

Lemma 1.6. Assume that statement (2) of the above theorem holds true. Then, the functions $\alpha_{t}$ and $\beta_{t}$ are $R\left(\cdot \mid X_{t}\right)$-integrable P-a.e. and

$\left\{\begin{array}{l}0<E_{R}\left(\alpha_{t} \beta_{t} \mid X_{t}\right)=E_{R}\left(\alpha_{t} \mid X_{t}\right) E_{R}\left(\beta_{t} \mid X_{t}\right), \quad P \text {-a.e. } \\ 0 \leq E_{R}\left(\alpha_{t} \beta_{t} \mid X_{t}\right)=\mathbf{1}_{\left\{E_{R}\left(\alpha_{t} \mid X_{t}\right) E_{R}\left(\beta_{t} \mid X_{t}\right)<+\infty\right\}} E_{R}\left(\alpha_{t} \mid X_{t}\right) E_{R}\left(\beta_{t} \mid X_{t}\right), \quad \text { R-a.e. }\end{array}\right.$

Proof. See $[33, \S 3]$.

Lemma 1.6 leads to

$P\left(A \cap B \mid X_{t}\right)=\frac{E_{R}\left(\alpha_{t} \beta_{t} \mathbf{1}_{A} \mathbf{1}_{B} \mid X_{t}\right)}{E_{R}\left(\alpha_{t} \beta_{t} \mid X_{t}\right)}=\frac{E_{R}\left(\alpha_{t} \mathbf{1}_{A} \mid X_{t}\right)}{E_{R}\left(\alpha_{t} \mid X_{t}\right)} \frac{E_{R}\left(\beta_{t} \mathbf{1}_{B} \mid X_{t}\right)}{E_{R}\left(\beta_{t} \mid X_{t}\right)}, P$-a.e. 
Choosing $A=\Omega$ or $B=\Omega$ in this formula, we obtain

$$
\begin{aligned}
& P\left(B \mid X_{t}\right)=E_{R}\left(\beta_{t} \mathbf{1}_{B} \mid X_{t}\right) / E_{R}\left(\beta_{t} \mid X_{t}\right), \\
& P\left(A \mid X_{t}\right)=E_{R}\left(\alpha_{t} \mathbf{1}_{A} \mid X_{t}\right) / E_{R}\left(\alpha_{t} \mid X_{t}\right) .
\end{aligned}
$$

This completes the proof of (1.5).

- Proof of $(1) \Rightarrow(2)$. Take a Markov measure $P$ with derivative $Z$ with respect to $R: d P=Z d R$. We denote by

$$
\begin{aligned}
Z_{t} & :=E_{R}\left(Z \mid X_{[0, t]}\right), Z_{t}^{*}:=E_{R}\left(Z \mid X_{[t, 1]}\right) \text { and } \\
\zeta_{t}(z) & :=E_{R}\left(Z \mid X_{t}=z\right)=\frac{d P_{t}}{d R_{t}}(z) .
\end{aligned}
$$

Remark that the last equality implies that $\zeta_{t}\left(X_{t}\right)>0, P$-a.e.,

$$
\zeta_{t}\left(X_{t}\right)=E_{R}\left(Z_{t} \mid X_{t}\right)=E_{R}\left(Z_{t}^{*} \mid X_{t}\right), \quad R \text {-a.e. }
$$

and that $\zeta_{t}\left(X_{t}\right)$ is $R$-integrable.

Fix three bounded nonnegative functions $f, g, h$ that are respectively $\mathcal{A}_{[0, t]}$, $\mathcal{A}_{t}$ and $\mathcal{A}_{[t, 1]}$ measurable. One obtains

$$
\begin{array}{rll}
E_{P}(f g h) & \stackrel{(\mathrm{i})}{=} & E_{P}\left[E_{P}\left(f \mid X_{t}\right) g E_{P}\left(h \mid X_{t}\right)\right] \\
& \stackrel{(\mathrm{ii})}{=} & E_{P}\left[\frac{E_{R}\left(f Z_{t} \mid X_{t}\right)}{E_{R}\left(Z_{t} \mid X_{t}\right)} g \frac{E_{R}\left(h Z_{t}^{*} \mid X_{t}\right)}{E_{R}\left(Z_{t}^{*} \mid X_{t}\right)}\right] \\
& \stackrel{(\mathrm{iii})}{=} & E_{P}\left[g \frac{E_{R}\left(f h Z_{t} Z_{t}^{*} \mid X_{t}\right)}{\zeta_{t}\left(X_{t}\right)^{2}}\right] \\
& \stackrel{(\mathrm{iv})}{=} & E_{P}\left[g E_{\widetilde{P}}\left(f h \mid X_{t}\right)\right]
\end{array}
$$

where we successively used in (i): the Markov property of $P$, in (iii): identity (1.6) and the Markov property of $R$ and in (iv), we introduce the measure

$$
\widetilde{P}:=1_{\left\{\zeta_{t}\left(X_{t}\right)>0\right\}} \frac{Z_{t} Z_{t}^{*}}{\zeta_{t}\left(X_{t}\right)} R
$$

From all these identities one deduces that

$$
P\left(\cdot \mid X_{t}\right)=\widetilde{P}\left(\cdot \mid X_{t}\right), \quad P \text {-a.e. }
$$

Define

$$
\left\{\begin{array}{l}
\alpha_{t}=\mathbf{1}_{\left\{\zeta_{t}\left(X_{t}\right)>0\right\}} Z_{t} / \zeta_{t}\left(X_{t}\right) \\
\beta_{t}=Z_{t}^{*}
\end{array}\right.
$$

Therefore (1.7) becomes

$$
\widetilde{P}=\alpha_{t} \beta_{t} R
$$

and

$$
E_{R}\left(\alpha_{t} \mid X_{t}\right)=\mathbf{1}_{\left\{\zeta_{t}\left(X_{t}\right)>0\right\}} \text { and } E_{R}\left(\beta_{t} \mid X_{t}\right)=\zeta_{t}\left(X_{t}\right) \text {. }
$$


In order to identify $P$ with $\widetilde{P}$, since (1.8) is satisfied, it is enough to show that their marginals at time $t$ are the same. Let us prove it.

$$
\begin{aligned}
\widetilde{P}_{t}(d z) & =E_{R}\left(\alpha_{t} \beta_{t} \mid X_{t}=z\right) R_{t}(d z) \\
& \stackrel{(i)}{=} E_{R}\left(\alpha_{t} \mid X_{t}=z\right) E_{R}\left(\beta_{t} \mid X_{t}=z\right) R_{t}(d z) \\
& =\zeta_{t}(z) R_{t}(d z)=P_{t}(d z)
\end{aligned}
$$

where the Markov property of $R$ is used at (i). This fact, together with (1.8), implies the equality $P=\widetilde{P}$. Eventually, since $Z_{t}$ is $\mathcal{A}_{[0, t]}$-measurable and $Z_{t}^{*}$ is $\mathcal{A}_{[t, 1]}$-measurable, $\alpha_{t}$ and $\beta_{t}$ are respectively $\mathcal{A}_{[0, t]}$ and $\mathcal{A}_{[t, 1]}$-measurable functions.

Example 1.7. In the extreme case where $\alpha_{t}$ is $\mathcal{A}_{0}$-measurable (that is $\alpha_{t}=$ $f_{0}\left(X_{0}\right)$ ) and $\beta_{t}$ is $\mathcal{A}_{1}$-measurable (that is $\beta_{t}=g_{1}\left(X_{1}\right)$ ), one obtains from the above theorem that any measure $P$ of the form

$$
P=f_{0}\left(X_{0}\right) g_{1}\left(X_{1}\right) R
$$

is Markov. This was remarked in [18, Thm.4.1] (Eq. (1) applied to $a=0$ and $b=1)$.

In Theorem 2.14 we will see that, under some restrictions on $R$, the measures of the form (1.10) are the only ones which are Markov in the class of all measures of the form $P=h\left(X_{0}, X_{1}\right) R$.

\subsection{A fundamental example: Bridges of a Markov measure}

Since we are interested in a time-symmetric description of path measures, it is reasonable to disintegrate them along their endpoint (initial and final) values. Any probability measure $P$ on $\Omega$ is a mixture of measures pinned at both times $t=0$ and $t=1$, i.e. a mixture of its own bridges:

$$
P=\int_{\mathcal{X}^{2}} P\left(\cdot \mid X_{0}=x, X_{1}=y\right) P_{01}(d x d y) .
$$

Since $\mathcal{X}^{2}$ is Polish, this disintegration is meaningful. Note however that the bridge $P\left(\cdot \mid X_{0}, X_{1}\right)$ is a priori only defined $P$-a.s.

To simplify the presentation of our results, we will consider path measures $P$ whose bridges can be constructed for all $x, y \in \mathcal{X}$ as a regular version of the family of conditional laws $\left(P\left(\cdot \mid X_{0}=x, X_{1}=y\right), x, y \in \mathcal{X}\right)$ and denote them by $\left(P^{x y}\right)_{x, y \in \mathcal{X}}$. See for instance $[29$, Ch. 6$]$ for a precise definition of a regular conditional distribution.

Remark that it is not easy to construct such an everywhere-defined version in a general non-Markov setting but this is done in several relevant situations: When $P$ is a Lévy process - see [29] and [40, Prop. 3.1], a right process - see [22], or a Feller process - see [6].

We recall the important property that pinning preserves the Markov property. 
Proposition 1.8. Let $P$ be a Markov measure whose bridges are defined everywhere. Then, for any $x, y \in \mathcal{X}$, the bridge $P^{x y}$ is also Markov.

Proof. Let $P$ be a Markov measure, $t$ be a time in $[0,1], A \in \mathcal{A}_{[0, t]}$ and $B \in \mathcal{A}_{[t, 1]}$ be two events. We have

$$
P\left(A \cap B \mid X_{0}, X_{t}, X_{1}\right)=P\left(A \mid X_{0}, X_{t}\right) P\left(B \mid X_{0}, X_{t}, X_{1}\right), \quad P \text {-a.e. }
$$

Indeed,

$$
\begin{aligned}
P\left(A \cap B \mid X_{0}, X_{t}, X_{1}\right) & =E\left[P\left(A \cap B \mid X_{0}, X_{[t, 1]}\right) \mid X_{0}, X_{t}, X_{1}\right] \\
& =E\left[\mathbf{1}_{B} P\left(A \mid X_{0}, X_{[t, 1]}\right) \mid X_{0}, X_{t}, X_{1}\right] \\
& =E\left[\mathbf{1}_{B} P\left(A \mid X_{0}, X_{t}\right) \mid X_{0}, X_{t}, X_{1}\right] \\
& =P\left(A \mid X_{0}, X_{t}\right) P\left(B \mid X_{0}, X_{t}, X_{1}\right) .
\end{aligned}
$$

Moreover, by Theorem 1.2- $\left(2^{*}\right), P\left(A \mid X_{0}, X_{t}\right)=P\left(A \mid X_{0}, X_{t}, X_{1}\right)$. Therefore

$$
P^{X_{0}, X_{1}}\left(A \cap B \mid X_{t}\right)=P^{X_{0}, X_{1}}\left(A \mid X_{t}\right) P^{X_{0}, X_{1}}\left(B \mid X_{t}\right), \quad P \text {-a.e. }
$$

which characterizes the Markov property of every bridge $P^{x y}$ via (1.1).

In the rest of the section, we will work in the following framework.

Assumptions (A). There exists a reference Markov measure $R$ satisfying the following requirements.

$\left(\mathrm{A}_{1}\right) \quad R$ admits a family of bridges which can be defined everywhere

$\left(\mathrm{A}_{2}\right)$ The transition probability kernels of $R$ admit a density, denoted by $r$, with respect to some $\sigma$-finite positive measure $m$ on $\mathcal{X}$ : For all $0 \leq s<t \leq 1$,

$$
\begin{aligned}
r(s, x ; t, y) & :=\frac{d r(s, x ; t, \cdot)}{d m}(y) \text { for } R_{s} \otimes m \text {-a.e. }(x, y) \\
\text { and } \quad r^{*}(s, x ; t, y) & :=\frac{d r^{*}(s, \cdot ; t, y)}{d m}(x) \text { for } m \otimes R_{t} \text {-a.e. }(x, y) .
\end{aligned}
$$

Therefore $R_{0}(d x)=\int r^{*}(0, x ; 1, y) R_{1}(d y) m(d x)=: r_{0}(x) m(d x)$ and similarly $R_{1}(d y)=: r_{1}(y) m(d y)$. This leads to

$$
R_{01}(d x d y)=r_{0}(x) m(d x) r(0, x ; 1, y) m(d y)=r_{1}(y) m(d y) r^{*}(0, x ; 1, y) m(d x),
$$

in such a way that the function $c$ defined for almost every $x$ and $y$ by

$$
c(x, y):=r_{0}(x) r(0, x ; 1, y)=r_{1}(y) r^{*}(0, x ; 1, y)
$$

is the density of the joint marginal $R_{01}(d x d y)$ with respect to $m \otimes m$.

Remark that $\left(\mathrm{A}_{2}\right)$ is not always satisfied. For instance, let us consider a Poisson process $R$ with a random initial law that admits a density on $\mathbb{R}$. At any time $s$, its marginal law also admits a density. But the support of the measure $r(s, x ; t, d y)$ is discrete and equal to $x+\mathbb{N}$. Therefore there does not exist any 
measure $m$ such that for a.e. $x, r(s, x ; t, d y) \ll m(d y)$. We will see at Example 1.12(ii) how to circumvent this obstacle.

Let us recall the general structural relation between the path measure $R$ and its bridges. In general, the bridges are not globally absolutely continuous with respect to $R$, but they are locally absolutely continuous with respect to $R$ in restriction to time interval $[s, t] \subset(0,1)$ away from the terminal times 0 and 1 .

Theorem 1.9. Consider a Markov measure $R$ satisfying Assumptions (A). For all $0<s \leq t<1$ and all $x, y \in \mathcal{X}$, the bridge $\left(R^{x y}\right)_{[s, t]}$ of $R$ restricted to $\mathcal{A}_{[s, t]}$ is dominated by $R_{[s, t]}$. Its density is given by

$$
\left(R^{x y}\right)_{[s, t]}=\frac{r^{*}\left(0, x ; s, X_{s}\right) r\left(t, X_{t} ; 1, y\right)}{c(x, y)} R_{[s, t]},
$$

where the function $c$ is defined by (1.13).

Proof. Let us show that

$$
c(x, y)=0 \Rightarrow r^{*}(0, x ; s, z) r\left(t, z^{\prime} ; 1, y\right)=0, \quad \forall\left(z, z^{\prime}\right), R_{s t^{-} \text {-a.e. }}
$$

On the one hand,

$$
R_{01}(d x d y)=c(x, y) m(d x) m(d y)
$$

and on the other hand, following Proposition 1.4,

$$
\begin{aligned}
R_{01}(d x d y) & =\int_{\mathcal{X}^{2}} R_{0, s, t, 1}\left(d x, d z, d z^{\prime}, d y\right) \\
& =\int_{\mathcal{X}^{2}} r^{*}(0, d x ; s, z) R_{s}(d z) r\left(s, z ; t, d z^{\prime}\right) r\left(t, z^{\prime} ; 1, d y\right) \\
& =\int_{\mathcal{X}^{2}} r^{*}(0, x ; s, z) r\left(s, z ; t, z^{\prime}\right) r\left(t, z^{\prime} ; 1, y\right) R_{s}(d z) m\left(d z^{\prime}\right) m(d x) m(d y) .
\end{aligned}
$$

Then

$$
c(x, y)=\int_{\mathcal{X}^{2}} r^{*}(0, x ; s, z) r\left(s, z ; t, z^{\prime}\right) r\left(t, z^{\prime} ; 1, y\right) R_{s}(d z) m\left(d z^{\prime}\right)
$$

and (1.15) holds. This allows us not to bother about dividing by zero.

For $R_{s t}$-a.e. $\left(z, z^{\prime}\right)$, the measure $r^{*}(0, d x ; s, z) r\left(t, z^{\prime} ; 1, d y\right)$ is dominated by $R_{01}(d x d y)$ and it satisfies

$$
r^{*}(0, d x ; s, z) r\left(t, z^{\prime} ; 1, d y\right)=\frac{r^{*}(0, x ; s, z) r\left(t, z^{\prime} ; 1, y\right)}{c(x, y)} R_{01}(d x d y) .
$$

Take two bounded measurable functions $f, g$ and an event $B \in \mathcal{A}_{[s, t]}$. Thus,

$$
\begin{aligned}
& E_{R}\left[f\left(X_{0}\right) \mathbf{1}_{B} g\left(X_{1}\right)\right] \\
& =E_{R}\left[\mathbf{1}_{B} E_{R}\left(f\left(X_{0}\right) \mid X_{[s, t]}\right) E_{R}\left(g\left(X_{1}\right) \mid X_{[s, t]}\right)\right] \\
& =E_{R}\left[\mathbf{1}_{B} E_{R}\left(f\left(X_{0}\right) \mid X_{s}\right) E_{R}\left(g\left(X_{1}\right) \mid X_{t}\right)\right]
\end{aligned}
$$




$$
\begin{aligned}
& =E_{R}\left[\mathbf{1}_{B} \int_{\mathcal{X}} f(x) r^{*}\left(0, d x ; s, X_{s}\right) \int_{\mathcal{X}} g(y) r\left(t, X_{t} ; 1, d y\right)\right] \\
& =E_{R}\left[\mathbf{1}_{B} \int_{\mathcal{X}^{2}} f(x) g(y) r^{*}\left(0, d x ; s, X_{s}\right) r\left(t, X_{t} ; 1, d y\right)\right] \\
& \stackrel{\vee}{=} E_{R}\left[\mathbf{1}_{B} \int_{\mathcal{X}^{2}} f(x) \frac{r^{*}\left(0, x ; s, X_{s}\right) r\left(t, X_{t} ; 1, y\right)}{c(x, y)} g(y) R_{01}(d x d y)\right] \\
& =\int_{\mathcal{X}^{2}} f(x) E_{R}\left[\mathbf{1}_{B} \frac{r^{*}\left(0, x ; s, X_{s}\right) r\left(t, X_{t} ; 1, y\right)}{c(x, y)}\right] g(y) R_{01}(d x d y),
\end{aligned}
$$

where we used (1.16) at the marked equality. This proves (1.14).

Corollary 1.10 (Decomposition of a bridge). Introducing $f_{s}(z):=r^{*}(0, x ; s, z)$ and $g_{t}\left(z^{\prime}\right)=: c(x, y)^{-1} r\left(t, z^{\prime} ; 1, y\right)$, (1.14) becomes

$$
\left(R^{x y}\right)_{[s, t]}=f_{s}\left(X_{s}\right) g_{t}\left(X_{t}\right) R_{[s, t]} .
$$

In particular, at each time $t \in(0,1)$, the one dimensional marginal of the bridge $R^{x y}$ is dominated by the marginal $R_{t}$ of the Markov measure $R$. It satisfies

$$
R_{t}^{x y}=f_{t}\left(X_{t}\right) g_{t}\left(X_{t}\right) R_{t} .
$$

One interprets (1.17) as a generalization of (1.10) on the time interval $[s, t]$ : the density of the bridge decomposes into a product of functions of the process at boundary times $s$ and $t$. This ensures its Markov property.

Naturally, both forward and backward dynamics of the bridge are directly related to the dynamics of the reference process with free boundary conditions.

Proposition 1.11. Let $R$ be a Markov measure.

(1) For any time $0<t<1$ and for any $(x, y)$, the bridge $R^{x y}$ of $R$, restricted to $\mathcal{A}_{[0, t]}$ is given by

$$
\left(R^{x y}\right)_{[0, t]}=\frac{r\left(t, X_{t} ; 1, y\right)}{r(0, x ; 1, y)} R_{[0, t]}\left(\cdot \mid X_{0}=x\right) .
$$

(2) Analogously, for any time $0<s<1$ and for any $(x, y)$, the bridge $R^{x y}$ of $R$ restricted to $\mathcal{A}_{[s, 1]}$ is given by

$$
\left(R^{x y}\right)_{[s, 1]}=\frac{r^{*}\left(0, x ; s, X_{s}\right)}{r^{*}(0, x ; 1, y)} R_{[s, 1]}\left(\cdot \mid X_{1}=y\right) .
$$

(3) The forward and backward transition probability kernels of $R^{x y}$ satisfy for all $0 \leq s<t \leq 1$ and $R_{s t}$-a.e. $\left(z, z^{\prime}\right)$,

$$
\begin{aligned}
& r^{x y}\left(s, z ; t, d z^{\prime}\right)=\mathbf{1}_{\{r(s, z ; 1, y)>0\}} \frac{r\left(s, z ; t, z^{\prime}\right) r\left(t, z^{\prime} ; 1, y\right)}{r(s, z ; 1, y)} m\left(d z^{\prime}\right) \\
& r_{*}^{x y}\left(s, d z ; t, z^{\prime}\right)=\mathbf{1}_{\left\{r^{*}\left(0, x ; t, z^{\prime}\right)>0\right\}} \frac{r^{*}(0, x ; s, z) r^{*}\left(s, z ; t, z^{\prime}\right)}{r^{*}\left(0, x ; t, z^{\prime}\right)} m(d z)
\end{aligned}
$$

with the conventions $r(1, z ; 1, y)=\mathbf{1}_{\{z=y\}}$ and $r^{*}(0, x ; 0, z)=\mathbf{1}_{\{z=x\}}$. 
Proof. • Proof of (1). Define $P^{\widetilde{x y}}:=\frac{r\left(t, X_{t} ; 1, y\right)}{r(0, x ; 1, y)} R_{[0, t]}\left(\cdot \mid X_{0}=x\right)$ and take a bounded nonnegative map $f$ and an event $B \in \mathcal{A}_{[0, t]}$. Then,

$$
\begin{aligned}
E_{R}\left(\widetilde{P^{x X_{1}}}(B) f\left(X_{1}\right) \mid X_{0}=x\right) & =\int_{\mathcal{X}} r(0, x ; 1, y) P^{\widetilde{x y}}(B) f(y) m(d y) \\
& =\int_{\mathcal{X}} E_{R}\left[\mathbf{1}_{B} r\left(t, X_{t} ; 1, y\right) f(y) \mid X_{0}=x\right] m(d y) \\
& =E_{R}\left[\mathbf{1}_{B} \int_{\mathcal{X}} r\left(t, X_{t} ; 1, d y\right) f(y) m(d y) \mid X_{0}=x\right] \\
& =E_{R}\left[\mathbf{1}_{B} E_{R}\left(f\left(X_{1}\right) \mid X_{t}\right) \mid X_{0}=x\right] \\
& =E_{R}\left[\mathbf{1}_{B} E_{R}\left(f\left(X_{1}\right) \mid X_{[0, t]}\right) \mid X_{0}=x\right] \\
& =E_{R}\left[\mathbf{1}_{B} f\left(X_{1}\right) \mid X_{0}=x\right] \\
& =E_{R}\left[R^{x X_{1}}(B) f\left(X_{1}\right) \mid X_{0}=x\right]
\end{aligned}
$$

which proves (1.18).

- Proof of (2). It is analogous to (1).

- Proof of (3). It is a direct corollary of (1) and (2).

Examples 1.12. Let us provide examples of several kinds of bridges.

(i) The first example is standard. Let $R=\mathbf{W}$ be a Wiener measure on the set of real-valued continuous paths on $[0,1]$, with fixed initial condition $x \in \mathbb{R}$. Assumption $\left(A_{1}\right)$ is satisfied since Brownian bridges can be constructed for any $x, y \in \mathbb{R}$ (as Paul Lévy already proposed). Assumption $\left(\mathrm{A}_{2}\right)$ is satisfied with $m(d x)=d x$. Then the forward and backward transition probability densities are given, for any $s \leq t, x, y \in \mathbb{R}$, by:

$$
\begin{aligned}
r(s, z ; t, y) & =\frac{1}{\sqrt{2 \pi(t-s)}} e^{-\frac{(y-z)^{2}}{2(t-s)}} \\
r^{*}(s, z ; t, y) & =\frac{r(0, x ; s, z) r(s, z ; t, y)}{r(0, x ; t, y)} .
\end{aligned}
$$

Therefore, due to (1.18), the Brownian bridge restricted to $\mathcal{A}_{[0, t]}$ satisfies

$$
\left(\mathbf{W}^{x, y}\right)_{[0, t]}=\frac{1}{\sqrt{1-t}} e^{-\left(\frac{\left(y-X_{t}\right)^{2}}{2(1-t)}-\frac{(y-x)^{2}}{2}\right)} \mathbf{W}_{[0, t]}
$$

(ii) Let $\mathbf{P}$ be the law of a Poisson process with values in the set of càdlàg step functions with positive unit jumps. Poisson bridges can be constructed for all $x, y \in \mathbb{R}$ such that $y-x \in \mathbb{N}$.

Now suppose that $X_{0}$ under $\mathbf{P}$ is random, real-valued and admits a density: $\mathbf{P}_{0}(d x)=r_{0}(x) d x$ on $\mathbb{R}$. As already remarked, such a process does not satisfy Assumption $\left(\mathrm{A}_{2}\right)$. However its dynamics is space- (and time-) homogeneous:

$$
r(s, x ; t, d y)=\delta_{x} * r(0,0 ; t-s, d y)
$$


and the transition kernel $r(0,0 ; u, d y)$ admits a Poissonian density $r$ with respect to the counting measure $m$ on $\mathbb{N}$ :

$$
r(0,0 ; u, d y)=r(u, y) m(d y) \quad \text { where } \quad r(u, n)=e^{-u} u^{n} / n ! .
$$

Therefore the proof of (1.18) can be generalized to this case, since one exhibits the density of the bridge, on the time interval $[0, t]$, of the Poisson process between 0 and $n$ with respect to the standard Poisson process starting in 0 . Then, the density on the time interval $[0, t]$ of the Poisson process pinned at $x$ and $y$ with respect to the Poisson process starting from $x$ satisfies for $\mathbf{P}_{0}$-a.e. $x$ and $y \in x+\mathbb{N}$,

$$
\begin{aligned}
\left(\mathbf{P}^{x y}\right)_{[0, t]} & =\frac{r\left(1-t, y-X_{t}\right)}{r(1, y-x)} \mathbf{P}_{[0, t]}\left(\cdot \mid X_{0}=x\right) \\
& =e^{t}(1-t)^{y-X_{t}} \frac{(y-x) !}{\left(y-X_{t}\right) !} \mathbf{P}_{[0, t]}\left(\cdot \mid X_{0}=x\right) .
\end{aligned}
$$

(iii) Let $\mathbf{C}$ be the law of a Cauchy process on $\Omega$. A regular version of Cauchy bridges can be constructed for all $x, y \in \mathbb{R}$, see [6]. The forward transition density $r(s, x ; t, y)$ is given, for each $x, y \in \mathbb{R}$, by the Cauchy law with parameter $t-s$ :

$$
r(s, x ; t, y)=\frac{t-s}{\pi\left((t-s)^{2}+(y-x)^{2}\right)}
$$

and for $\mathbf{C}_{0^{-}}$-almost all $x$,

$$
\left(\mathbf{C}^{x y}\right)_{[0, t]}=(1-t) \frac{1+(y-x)^{2}}{(1-t)^{2}+\left(y-X_{t}\right)^{2}} \quad \mathbf{C}_{[0, t]}\left(\cdot \mid X_{0}=x\right) .
$$

The computation of the density of the bridge on the time interval $[s, 1]$ follows the same schema, using the backward transition density and the initial value $\mathbf{C}_{0}$. One could also consider the reversible situation, corresponding to $\mathbf{C}_{0}(d x)=d x$. This reversible measure cannot be normalized but the present techniques remain valid for $\sigma$-finite measures, see [33].

(iv) Several other examples of Lévy bridges can be found in [40].

\section{Reciprocal measures}

We now enlarge our framework to the class of reciprocal measures. They are not necessarily Markov but they enjoy a more general time-symmetry which justifies their relevance in the study of quantum mechanical systems, see [38, 37, 10]. The dynamical properties of reciprocal diffusions were elaborated many years after their introduction by Bernstein, see $[26,55]$ and Section 3.1 for further details.

In fact reciprocal processes can be viewed as one-dimensional Markov random fields indexed by a compact time interval, here $[0,1]$, see Eq. (2.2). When the 


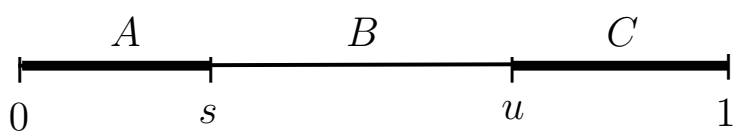

FIG 1 .

time parameter belongs to an unbounded interval, the issues are close to those that were encountered in the setting of Gibbs measures on the one-dimensional time space and quasi-invariant measures on the path space. They were extensively studied in the seventies in the context of Euclidean quantum field theory, see $[15,44,18]$ and references therein. A more recent result on existence and uniqueness of Gibbs measures for suitable potentials relative to Brownian motion was proved in [39].

\subsection{Definition and basic properties}

Let us begin with the definition.

Definition 2.1 (Reciprocal measure). A probability measure $P$ on $\Omega$ is called reciprocal if for any times $s \leq u$ in $[0,1]$ and for any events $A \in \mathcal{A}_{[0, s]}, B \in$ $\mathcal{A}_{[s, u]}, C \in \mathcal{A}_{[u, 1]}$, see Figure 1,

$$
P\left(A \cap B \cap C \mid X_{s}, X_{u}\right)=P\left(A \cap C \mid X_{s}, X_{u}\right) P\left(B \mid X_{s}, X_{u}\right) \quad P \text {-a.e. }
$$

The above property, which was first formalized by Jamison in [27], states that under $P$, given the knowledge of the canonical process at both times $s$ and $u$, the events "inside" $[s, u]$ and those "outside" $(s, u)$ are conditionally independent. It is clearly time-symmetric.

Paralleling Theorem 1.2, we present at Theorem 2.2 several characterizations of the reciprocal property. For the ease of the reader, we sketch its elementary proof.

Identity (2.2) states that a reciprocal measure is indeed a Markov field indexed by time, seen as a one-dimensional continuous parameter process. It means that conditioning an event depending on the inside data $X_{[s, u]}$ by the knowledge of the outside data $X_{[0, s] \cup[u, 1]}$ amounts to simply conditioning it by the knowledge of the boundary data $\left(X_{s}, X_{u}\right)$.

Theorem 2.2. Let $P$ be a probability measure on $\Omega$. Then the following assertions are equivalent:

(1) The measure $P$ is reciprocal.

(1*) The time-reversed measure $P^{*}$ is reciprocal (assuming (0.1)).

(2) For all $0 \leq s \leq u \leq 1$ and all sets $B \in \mathcal{A}_{[s, u]}$,

$$
P\left(B \mid X_{[0, s]}, X_{[u, 1]}\right)=P\left(B \mid X_{s}, X_{u}\right) \quad P \text {-a.e. }
$$




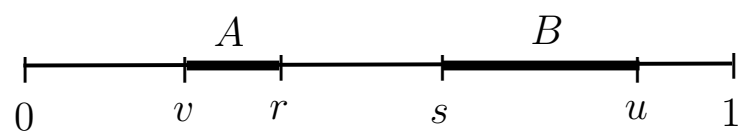

FIG 2.

(3) For all $0 \leq v \leq r \leq s \leq u \leq 1$ and all sets $A \in \mathcal{A}_{[v, r]}, B \in \mathcal{A}_{[s, u]}$, see Figure 2,

$$
P\left(A \cap B \mid X_{[0, v]}, X_{[r, s]}, X_{[u, 1]}\right)=P\left(A \mid X_{v}, X_{r}\right) P\left(B \mid X_{s}, X_{u}\right) \quad P \text {-a.e. }
$$

Proof. • Proof of $(1) \Leftrightarrow\left(1^{*}\right)$. Straightforward.

- Proof of $(1) \Rightarrow(2)$. Let us take $B \in \mathcal{A}_{[s, u]} . P\left(B \mid X_{[0, s]}, X_{[u, 1]}\right)$ is the unique random variable $\mathcal{A}_{[0, s]} \vee \mathcal{A}_{[u, 1]}$-measurable such that, for all $A \in \mathcal{A}_{[0, s]}$ and $C \in \mathcal{A}_{[u, 1]}$,

$$
P(A \cap B \cap C)=E\left[\mathbf{1}_{A} \mathbf{1}_{C} P\left(B \mid X_{[0, s]}, X_{[u, 1]}\right)\right] .
$$

But, due to (2.1), one has

$$
\begin{aligned}
P(A \cap B \cap C) & =E\left(P\left(A \cap B \cap C \mid X_{s}, X_{u}\right)\right) \\
& =E\left[P\left(A \cap C \mid X_{s}, X_{u}\right) P\left(B \mid X_{s}, X_{u}\right)\right] \\
& =E\left[E\left(\mathbf{1}_{A} \mathbf{1}_{C} P\left(B \mid X_{s}, X_{u}\right) \mid X_{s}, X_{u}\right)\right] \\
& =E\left[\mathbf{1}_{A} \mathbf{1}_{C} P\left(B \mid X_{s}, X_{u}\right)\right] .
\end{aligned}
$$

This implies (2).

- Proof of $(2) \Rightarrow(1)$. Let us take $0 \leq s \leq u \leq 1, A \in \mathcal{A}_{[0, s]}$, $B \in \mathcal{A}_{[s, u]}, C \in \mathcal{A}_{[u, 1]}$ and $f, g$ some measurable nonnegative functions. By definition,

$$
E\left[\mathbf{1}_{A} \mathbf{1}_{B} \mathbf{1}_{C} f\left(X_{s}\right) g\left(X_{u}\right)\right]=E\left[P\left(A \cap B \cap C \mid X_{s}, X_{u}\right) f\left(X_{s}\right) g\left(X_{u}\right)\right]
$$

holds. But,

$$
\begin{aligned}
E\left[\mathbf{1}_{A} \mathbf{1}_{B} \mathbf{1}_{C} f\left(X_{s}\right) g\left(X_{u}\right)\right] & =E\left[E\left(\mathbf{1}_{A} \mathbf{1}_{B} \mathbf{1}_{C} f\left(X_{s}\right) g\left(X_{u}\right) \mid X_{[0, s]}, X_{[u, 1]}\right)\right] \\
& =E\left[\mathbf{1}_{A} \mathbf{1}_{C} P\left(B \mid X_{s}, X_{u}\right) f\left(X_{s}\right) g\left(X_{u}\right)\right] \\
& =E\left[P\left(A \cap C \mid X_{s}, X_{u}\right) P\left(B \mid X_{s}, X_{u}\right) f\left(X_{s}\right) g\left(X_{u}\right)\right] .
\end{aligned}
$$

Therefore

$$
P\left(A \cap B \cap C \mid X_{s}, X_{u}\right)=P\left(A \cap C \mid X_{s}, X_{u}\right) P\left(B \mid X_{s}, X_{u}\right) .
$$

- Proof of $(2) \Rightarrow(3)$. Take $A \in \mathcal{A}_{[v, r]}$ and $B \in \mathcal{A}_{[s, u]}$. Then

$$
\begin{aligned}
& P\left(A \cap B \mid X_{[0, v]}, X_{[r, s]}, X_{[u, 1]}\right) \\
= & E\left[P\left(A \cap B \mid X_{[0, v]}, X_{[r, 1]}\right) \mid X_{[0, v]}, X_{[r, s]}, X_{[u, 1]}\right]
\end{aligned}
$$




$$
\begin{aligned}
& \stackrel{\vee}{=}\left[P\left(A \mid X_{v}, X_{r}\right) \mathbf{1}_{B} \mid X_{[0, v]}, X_{[r, s]}, X_{[u, 1]}\right] \\
& =E\left[E\left(P\left(A \mid X_{v}, X_{r}\right) \mathbf{1}_{B} \mid X_{[0, s]}, X_{[u, 1]}\right) \mid X_{[0, v]}, X_{[r, s]}, X_{[u, 1]}\right] \\
& \stackrel{\checkmark}{=} E\left[P\left(A \mid X_{v}, X_{r}\right) P\left(B \mid X_{s}, X_{u}\right) \mid X_{[0, v]}, X_{[r, s]}, X_{[u, 1]}\right] \\
& =P\left(A \mid X_{v}, X_{r}\right) P\left(B \mid X_{s}, X_{u}\right)
\end{aligned}
$$

where we used assumption (2) at the $\checkmark$-marked equalities.

- Proof of $(3) \Rightarrow(2)$. It is enough to take $A=\Omega$ and $v=t=s$.

For a measure, being Markov is stronger than being reciprocal. This was noticed by Jamison in $[26,27]$.

Proposition 2.3. Any Markov measure is reciprocal, but the converse is false.

Proof. Take $P$ a Markov measure, $0 \leq s \leq u \leq 1$ and $A \in \mathcal{A}_{[0, s]}, B \in \mathcal{A}_{[s, u]}$ and $C \in \mathcal{A}_{[u, 1]}$. The following holds:

$$
\begin{aligned}
& P(A \cap B \cap C) \quad=\quad E\left[P\left(A \cap B \cap C \mid X_{[s, u]}\right)\right] \\
& \stackrel{(i)}{=} \quad E\left[P\left(A \mid X_{s}\right) \mathbf{1}_{B} P\left(C \mid X_{u}\right)\right] \\
& =E\left[P\left(A \mid X_{s}\right) P\left(B \mid X_{s}, X_{u}\right) P\left(C \mid X_{u}\right)\right] \\
& \stackrel{(i i)}{=} E\left[P\left(A \mid X_{s}\right) P\left(B \mid X_{s}, X_{u}\right) P\left(C \mid X_{[0, u]}\right)\right] \\
& =E\left[P\left(A \mid X_{s}\right) P\left(B \mid X_{s}, X_{u}\right) \mathbf{1}_{C}\right] \\
& \stackrel{(\text { iii })}{=} E\left[P\left(A \mid X_{[s, 1]}\right) P\left(B \mid X_{s}, X_{u}\right) \mathbf{1}_{C}\right] \\
& =E\left[\mathbf{1}_{A} P\left(B \mid X_{s}, X_{u}\right) \mathbf{1}_{C}\right]
\end{aligned}
$$

Equality $(i)$ is due to Theorem 1.2(3). Use the Markov property to prove (ii) and (iii). Therefore (2.2) holds.

Examples 2.4 and 2.6-(ii) below provide examples of reciprocal measures which are not Markov. Other counterexamples will be given in Section 2.7, where we point out reciprocal processes whose endpoint marginal laws do not have the required structure which characterizes the Markov property.

Example 2.4 (Reciprocal measures on a loop space). Let us mention the following class of reciprocal - but not Markov - measures. Take a Markov measure $R$ whose bridges are defined everywhere and $m$ any probability measure on $\mathcal{X}$. Then

$$
P_{\text {loop }}:=\int_{\mathcal{X}} R^{x x} m(d x)
$$

is a measure that is concentrated on loops, i.e. paths such that $X_{0}=X_{1}$ almost surely, with both initial and final marginal laws equal to $m$. One can see this path measure as describing a periodic random process. Due to Proposition 2.7, $P_{\text {loop }}$ is reciprocal with the mixing measure $\pi(d x d y)=m(d x) \delta_{x}(d y)$.

To see that the Markov property breaks down in general, take $R^{x x}$ to be the usual Brownian bridge on $\mathbb{R}$ between $x$ and $x$, choose $m=\left(\delta_{-1}+\delta_{+1}\right) / 2$ and pick 
any intermediate time $0<t<1$. We have $P_{\text {loop }}\left(X_{1} \geq 0 \mid X_{[0, t]}\right)=\mathbf{1}_{\left\{X_{0}=+1\right\}}$, while

$$
P_{\text {loop }}\left(X_{1} \geq 0 \mid X_{t}\right)=P_{\text {loop }}\left(X_{0}=+1 \mid X_{t}\right) .
$$

In particular when $t=1 / 2$, symmetry considerations lead us to $P_{\text {loop }}\left(X_{0}=\right.$ $\left.+1 \mid X_{1 / 2}\right)=1 / 2$, implying that $P_{\text {loop }}\left(X_{1} \geq 0 \mid X_{1 / 2}\right)=1 / 2 \neq \mathbf{1}_{\left\{X_{0}=+1\right\}}=$ $P_{\text {loop }}\left(X_{1} \geq 0 \mid X_{[0, t]}\right)$.

We will describe in a short while the typical structure of reciprocal measures.

\subsection{Pinning leads back to the Markov property}

Proposition 1.8 states the stability of the Markov property by pinning. Similarly, Jamison pointed out in [27, Lemma 1.4] the remarkable property that pinning a reciprocal measure, not only preserves its reciprocal property, but also transforms it into a Markov one.

Proposition 2.5. Let $P$ be a reciprocal measure. If either $X_{0}$ or $X_{1}$ is a.s. constant, then $P$ is a Markov measure. In particular any bridge $P\left(\cdot \mid X_{0}, X_{1}\right)$, defined $P_{01}$-almost surely, is a Markov measure.

Proof. Suppose $X_{1}$ is $P$-a.s. constant and take $0 \leq s \leq u \leq 1$. For any bounded measurable function $f$

$$
\begin{aligned}
E_{P}\left(f\left(X_{u}\right) \mid X_{[0, s]}\right) & =E_{P}\left(f\left(X_{u}\right) \mid X_{[0, s]}, X_{1}\right)=E_{P}\left(f\left(X_{u}\right) \mid X_{s}, X_{1}\right) \\
& =E_{P}\left(f\left(X_{u}\right) \mid X_{s}\right)
\end{aligned}
$$

which characterises the Markov property of $P$ thanks Theorem 1.2 (2). The case where $X_{0}$ is $P$-a.s. constant is solved by time reversal.

\subsection{Mixing properly preserves the reciprocal property}

To complement the previous subsection we analyse in which way mixing (pinned) measures perturbs their reciprocal and/or Markov properties.

Mixing Markov measures sometimes preserves the Markov property, but this is far from being the rule. Similarly, mixing reciprocal measures sometimes results in a reciprocal measure, but not always. The following examples illustrate these assertions. Moreover, we construct in (ii) an example of a reciprocal measure which is not Markov.

Examples 2.6 (Various mixtures of deterministic paths). Let $\mathcal{X}=\{a, b, c\}$ be a state space with three elements. We denote by $\delta_{w}, w \in \Omega$, the Dirac measure at the path $w$. Any $\delta_{w}$ is Markov since the path $w$ is deterministic.

(i) One denotes by acb $\in \Omega$ the following path $w$ :

$$
\operatorname{acb}(t):=\mathbf{1}_{[0,1 / 3)}(t) \mathrm{a}+\mathbf{1}_{[1 / 3,2 / 3)}(t) c+\mathbf{1}_{[2 / 3,1]}(t) \text { b. }
$$


Similar notations are used for paths that only jump at times $1 / 3$ or $2 / 3$. The path measure

$$
P=\frac{1}{4}\left(\delta_{\mathrm{abc}}+\delta_{\mathrm{aba}}+\delta_{\mathrm{cba}}+\delta_{\mathrm{cbc}}\right)
$$

is the uniform mixture of deterministic Markov paths and is Markov too. Indeed $P_{0}=\frac{1}{2}\left(\delta_{\mathrm{a}}+\delta_{\mathrm{c}}\right)$ and the nontrivial transition measures which are given by

$$
P\left(X_{1 / 3}=\mathrm{b} \mid X_{0}=\mathrm{a}\right)=P\left(X_{1 / 3}=\mathrm{b} \mid X_{0}=\mathrm{c}\right)=1
$$

and

$$
\begin{aligned}
P\left(X_{2 / 3}=\mathrm{a} \mid X_{1 / 3}=\mathrm{b}, X_{0}=\mathrm{a}\right) & =P\left(X_{2 / 3}=\mathrm{a} \mid X_{1 / 3}=\mathrm{b}, X_{0}=\mathrm{c}\right) \\
& =P\left(X_{2 / 3}=\mathrm{c} \mid X_{1 / 3}=\mathrm{b}\right)=1 / 2
\end{aligned}
$$

entirely specify the dynamics of $P$.

(ii) The path measure

$$
P=\frac{1}{2}\left(\delta_{\mathrm{abc}}+\delta_{\mathrm{cba}}\right),
$$

is reciprocal but not Markov. It is reciprocal since each boundary condition determines the path. Nevertheless we observe that $P$ is not Markov since

$$
P\left(X_{1}=\mathrm{a} \mid X_{0}=\mathrm{a}, X_{1 / 3}=\mathrm{b}\right)=0
$$

while

$$
P\left(X_{1}=\mathrm{a} \mid X_{1 / 3}=\mathrm{b}\right)=1 / 2 .
$$

(iii) Now, we define paths with four states and three jumps at fixed times $1 / 4,1 / 2$ et $3 / 4$, such as

$$
\operatorname{abab}(t):=\mathbf{1}_{[0,1 / 4)}(t) a+\mathbf{1}_{[1 / 4,1 / 2)}(t) b+\mathbf{1}_{[1 / 2,3 / 4)}(t) a+\mathbf{1}_{[3 / 4,1]}(t) b .
$$

The path measure $P:=\frac{1}{2}\left(\delta_{\mathrm{abab}}+\delta_{\mathrm{cbcb}}\right)$, which is a mixture of reciprocal paths (they are deterministic) is not reciprocal anymore. Indeed

$$
P\left(X_{2 / 3}=\mathrm{a} \mid X_{[0,1 / 3]}, X_{[4 / 5,1]}\right)=\mathbf{1}_{\left\{X_{0}=\mathrm{a}\right\}}
$$

while

$$
P\left(X_{2 / 3}=\mathrm{a} \mid X_{1 / 3}, X_{4 / 5}\right)=P\left(X_{2 / 3}=\mathrm{a}\right)=1 / 2 .
$$

To avoid the pathology (iii) let us now mix only measures $R^{x y}$ obtained as bridges of some given reciprocal measure $R$.

Proposition 2.7. Let $R$ be a reciprocal measure such that the mapping $(x, y) \in$ $\mathcal{X}^{2} \mapsto R^{x y}$ is defined everywhere and measurable. Then, for any probability measure $\pi$ on $\mathcal{X}^{2}$, the path measure

$$
P(\cdot)=\int_{\mathcal{X}^{2}} R^{x y}(\cdot) \pi(d x d y)
$$

is reciprocal. Moreover, the bridges of $P$ coincide with those of $R, P$-a.e. 
Proof. Let us show (2.2) under $P$. Let $0 \leq s \leq t \leq 1, A \in \mathcal{A}_{[0, s]}, B \in \mathcal{A}_{[s, u]}$ and $C \in \mathcal{A}_{[u, 1]}$. Then,

$$
\begin{aligned}
E_{P}\left[\mathbf{1}_{A} P\left(B \mid X_{[0, s]}, X_{[u, 1]}\right) \mathbf{1}_{C}\right] & =P(A \cap B \cap C) \\
& =\int_{\mathcal{X}^{2}} R^{x y}(A \cap B \cap C) \pi(d x d y) \\
& \stackrel{\vee}{=} \int_{\mathcal{X}^{2}} E_{R^{x y}}\left[\mathbf{1}_{A} R\left(B \mid X_{s}, X_{t}\right) \mathbf{1}_{C}\right] \pi(d x d y) \\
& =E_{P}\left[\mathbf{1}_{A} R\left(B \mid X_{s}, X_{t}\right) \mathbf{1}_{C}\right]
\end{aligned}
$$

where the reciprocal property is used at the marked equality. Thus $P(B \mid$ $\left.X_{[0, s]}, X_{[t, 1]}\right)$ only depends on $\left(X_{s}, X_{t}\right)$ and

$$
P\left(B \mid X_{[0, s]}, X_{[t, 1]}\right)=R\left(B \mid X_{s}, X_{t}\right), P \text {-a.e., }
$$

which completes the proof.

Let us observe that this result does not contradict Example 2.6(iii). Indeed, $P$ was expressed as a mixture of its own bridges, but not as a mixture of bridges of a given reciprocal measure. It happens that there does not exist any reciprocal measure $R$ such that $\delta_{\mathrm{abab}}=R^{\mathrm{ab}}$ and $\delta_{\mathrm{cbcb}}=R^{\mathrm{cb}}$.

\subsection{Reciprocal class associated with a measure}

The previous proposition allows to construct classes of reciprocal measures based on some reference reciprocal measure by varying the way of mixing bridges. Therefore, we now recall the important concept of reciprocal class which appears (implicitly) in [27, §3], associated with a Markov reference measure $R$ satisfying Assumptions (A) at page 246.

Definition 2.8 (Reciprocal class associated with $R$ ). Suppose that $R$ is a reciprocal measure such that $(x, y) \in \mathcal{X}^{2} \mapsto R^{x y}$ is defined everywhere and measurable. The set of probability measures on $\Omega$ defined by

$$
\mathfrak{R}(R):=\left\{P=\int_{\mathcal{X}^{2}} R^{x y}(\cdot) \pi(d x d y) ; \pi \text { probability measure on } \mathcal{X}^{2}\right\}
$$

is called the reciprocal class associated with $R$.

In the case of a discrete state space $\mathcal{X}$, the hypothesis on $R$ becomes unnecessary. One should only make sure that the support of the mixing measure $\pi$ is included in the support of $R_{01}$, in such a way that (2.3) makes sense.

In the particular case where $R$ is a Brownian diffusion defined on the space of continuous paths, the class $\mathfrak{R}(R)$ can be characterized by two functions of the drift of $R$, called reciprocal invariants. This was conjectured by Krener in [31] and proved by Clark in [11, Theorem 1]. See also [47] and [17] for their role in a second order stochastic differential equation satisfied by the reciprocal class. Thereafter, Thieullen and the second author derived an integration by parts formula on the path space that is expressed in terms of the reciprocal 
invariants of the Brownian diffusion $R$ and that fully characterises the associated reciprocal class. See [42] for one-dimensional diffusion processes and [43] for the multidimensional case.

When $R$ is a counting process (i.e. $\mathcal{X}=\mathbb{N}$ ), Murr provides a description of a reciprocal invariant associated with $\mathfrak{R}(R)$, as well as a characterisation of the reciprocal class through a duality formula, see [36, 14]. An extension of this work for compound Poisson processes is done in [12], and to more general processes on graphs in [13].

For a recent review on stochastic analysis methods to characterize reciprocal classes, see [41].

\subsection{Time reversal and reciprocal classes}

We already saw in Theorem 2.2 that a path measure is reciprocal if and only if its time-reversed is reciprocal too. We now precise what is the image of a reciprocal class by time reversal. We give the proof, even if it looks rather natural.

Proposition 2.9. Let $R$ be a reciprocal measure as in Definition 2.8. Then

$$
P \in \mathfrak{R}(R) \Longleftrightarrow P^{*} \in \mathfrak{R}\left(R^{*}\right) .
$$

We first prove the following auxiliary lemma.

Lemma 2.10. Let $P$ be a probability measure on $\Omega$.

(a) Consider the diagram $\Omega \stackrel{\Phi}{\rightarrow} \Phi(\Omega) \stackrel{\theta}{\rightarrow} \mathcal{Y}$ where the mentioned sets and mappings are measurable. Then, for any bounded measurable function $f$ : $\Phi(\Omega) \rightarrow \mathbb{R}$, we have

$$
E_{\Phi_{\#} P}(f \mid \theta)=\alpha(\theta)
$$

with $\alpha(y):=E_{P}(f(\Phi) \mid \theta(\Phi)=y)$.

(b) Consider the diagram $\mathcal{Y} \stackrel{\theta}{\leftarrow} \Omega \stackrel{\Phi}{\rightarrow} \Omega$ where the mentioned sets and mappings are measurable. Suppose that $\Phi$ is one-to-one with measurable inverse $\Phi^{-1}$. Then,

$$
\Phi_{\#}[P(\cdot \mid \theta=y)]=\left[\Phi_{\#} P\right]\left(\cdot \mid \theta \circ \Phi^{-1}=y\right), \quad y \in \mathcal{Y} .
$$

Proof. • Proof of (a). For any bounded measurable function $u: \mathcal{Y} \rightarrow \mathbb{R}$,

$$
\begin{aligned}
E_{\Phi_{\#} P}\left[E_{\Phi_{\#} P}(f \mid \theta) u(\theta)\right] & =E_{\Phi_{\#} P}(f u(\theta))=E_{P}[f(\Phi) u(\theta(\Phi))] \\
& =E_{P}\left[E_{P}(f(\Phi) \mid \theta(\Phi)) u(\theta(\Phi))\right]=E_{\Phi_{\#} P}(\alpha(\theta) u(\theta))
\end{aligned}
$$

- Proof of (b). We add a bounded measurable function $u$ to the diagram: $\mathcal{Y} \stackrel{\theta}{\leftarrow} \Omega \stackrel{\Phi}{\rightarrow} \Omega \stackrel{u}{\rightarrow} \mathbb{R}$ and compute, for $y \in \mathcal{Y}$,

$$
\begin{aligned}
E_{\Phi_{\#} P(\cdot \mid \theta=y)}(u) & =E_{P}[u(\Phi) \mid \theta=y] \\
& =E_{P}\left[u(\Phi) \mid \theta \circ \Phi^{-1} \circ \Phi=y\right] \stackrel{(i)}{=} E_{\Phi_{\#} P}\left(u \mid \theta \circ \Phi^{-1}=y\right)
\end{aligned}
$$

where equality (i) is a consequence of the above result (a). 
Proof of Proposition 2.9. In particular Lemma 2.10-(b) implies that

$$
\left(R^{x y}\right)^{*}=\left(R^{*}\right)^{y x}, \quad \text { for } R_{01} \text {-a.e. } x, y \in \mathcal{X} .
$$

Let $P \in \mathfrak{R}(R)$, then $P(\cdot)=\int_{\mathcal{X}^{2}} R^{x y}(\cdot) P_{01}(d x d y)$. We now compute the integral of a function $u$ under $P^{*}$ :

$$
\begin{aligned}
E_{P^{*}}[u(X)] & =E_{P}\left[u\left(X^{*}\right)\right]=\int_{\mathcal{X}^{2}} E_{\left(R^{x y}\right)^{*}}(u) P_{01}(d x d y) \\
& \stackrel{(2.4)}{=} \int_{\mathcal{X}^{2}} E_{\left(R^{*}\right)^{y x}}(u) P_{01}(d x d y)=\int_{\mathcal{X}^{2}} E_{\left(R^{*}\right)^{x y}}(u)\left(P^{*}\right)_{01}(d x d y) .
\end{aligned}
$$

This means that $P^{*}(\cdot)=\int_{\mathcal{X}^{2}}\left(R^{*}\right)^{x y}(\cdot)\left(P^{*}\right)_{01}(d x d y)$, completing the proof of Proposition 2.9.

\subsection{Reciprocal subclass of dominated measures}

To make precise our structural analysis of reciprocal measures, we introduce a slightly smaller family of measures than the reciprocal class. This subclass only contains measures which are dominated by the reference measure $R$.

Definition 2.11. Suppose that $R$ is a reciprocal measure as in Definition 2.8. We define the following set of probability measures on $\Omega$ :

$$
\mathfrak{R}_{a c}(R):=\left\{P: P=\int_{\mathcal{X}^{2}} R^{x y} \pi(d x d y) ; \pi \in \operatorname{Proba}\left(\mathcal{X}^{2}\right), \pi \ll R_{01}\right\} \subset \mathfrak{R}(R) .
$$

Remarks 2.12 (about this definition).

(a) Due to Proposition 2.7, we notice that any element of $\mathfrak{R}_{a c}(R)$ is reciprocal.

(b) We write $P \prec R$ when $P$ disintegrates as in (2.5). Note that the relation $\prec$ is transitive. But it is not symmetric; this lack of symmetry arises when the marginal laws at time 0 and 1 are not equivalent in the sense of measure theory. Therefore $\mathfrak{R}_{a c}(R)$ is not an equivalence class. If one wants to define a genuine equivalence relation $\sim$ between measures on $\Omega$ one should assume that marginal laws at time 0 and 1 are equivalent. Then $P \sim R$ if and only if $P \prec R$ and $R \prec P$.

As noticed in [18] Proposition 3.5, elements of $\mathfrak{R}_{a c}(R)$ have a simple structure.

Theorem 2.13. Each measure $P$ in $\mathfrak{R}_{a c}(R)$ is absolutely continuous with respect to $R$ and satisfies

$$
P=\frac{d \pi}{d R_{01}}\left(X_{0}, X_{1}\right) R
$$

Conversely, if $P$ is a path measure defined by

$$
P=h\left(X_{0}, X_{1}\right) R
$$

for some nonnegative measurable function $h$ on $\mathcal{X}^{2}$, then $P \in \mathfrak{R}_{a c}(R)$ and more precisely, $P$ is a $\pi$-mixture of bridges of $R$ with $\pi(d x d y):=h(x, y) R_{01}(d x d y)$. 
Proof. Let $P \in \mathfrak{R}_{a c}(R)$ and $f$ any nonnegative bounded function. Due to Definition (2.5),

$$
\begin{aligned}
E_{P}(f) & =\int_{\mathcal{X}^{2}} E_{R}\left(f \mid X_{0}=x, X_{1}=y\right) \frac{d \pi}{d R_{01}}(x, y) R_{01}(d x d y) \\
& =E_{R}\left(f \frac{d \pi}{d R_{01}}\left(X_{0}, X_{1}\right)\right),
\end{aligned}
$$

which proves the first assertion. For the second assertion, note that

$$
P(\cdot)=\int_{\mathcal{X}^{2}} P^{x y}(\cdot) \pi(d x d y)=\int_{\mathcal{X}^{2}} h(x, y) R^{x y}(\cdot) R_{01}(d x d y) .
$$

This completes the proof of the theorem.

The specific structure of $P$ which appears in (2.6) can be regarded as a timesymmetric version of the $h$-transform introduced by Doob in [20]:

$$
P\left(\cdot \mid X_{0}=x\right)=\frac{h\left(x, X_{1}\right)}{c_{x}} R\left(\cdot \mid X_{0}=x\right) \text { for } R_{0} \text {-a.e. } x \in \mathcal{X}
$$

and, in a symmetric way,

$$
P\left(\cdot \mid X_{1}=y\right)=\frac{h\left(X_{0}, y\right)}{C_{y}} R\left(\cdot \mid X_{1}=y\right) \text { for } R_{1} \text {-a.e. } y \in \mathcal{X}
$$

\subsection{Markov measures of a reciprocal class}

Since the Markov property is more restrictive than the reciprocal property, it is interesting to describe the subset of $\mathfrak{R}_{a c}(R)$ composed by the Markov measures. In other words, one is looking for the specific mixtures of measures which preserve Markov property.

If a measure in $\mathfrak{R}_{a c}(R)$ admits a density with respect to $R$ which is decomposable into a product as in (1.10), then it is Markov. Indeed this property is (almost) characteristic as will be stated below in Theorem 2.14. The first (partial) version of Theorem 2.14 can be found in [27, Thm. 3.1] when $R$ admits a strictly positive transition density: $P \in \mathfrak{R}(R)$ if and only if there exist two measures $\nu_{0}$ and $\nu_{1}$ on $\mathcal{X}$ such that

$$
P_{01}(d x d y)=r(0, x ; 1, y) \nu_{0}(d x) \nu_{1}(d y) .
$$

Jamison $[28$, p. 324] commented on this structure as that of an " $h$-path process in the sense of Doob". In the general framework of Markov field this result was proved in [18, Thm.4.1].

Our statement emphasizes the role of condition (2.8) which, up to our knowledge, comes out for the first time.

Theorem 2.14. Let $R$ and $P$ be two probability measures on $\Omega$ and suppose that $R$ is Markov. Consider the following assertions: 
(1) The measure $P$ belongs to $\mathfrak{R}_{a c}(R)$ and is Markov.

(2) There exist two measurable nonnegative functions $f_{0}$ and $g_{1}$ such that

$$
\frac{d P}{d R}=f_{0}\left(X_{0}\right) g_{1}\left(X_{1}\right), \quad R \text {-a.e. }
$$

Then, (2) implies assertion (1).

If we suppose moreover that there exists $0<t_{0}<1$ and a measurable subset $\mathcal{X}_{o} \subset \mathcal{X}$ such that $R_{t_{0}}\left(\mathcal{X}_{o}\right)>0$ and for all $z \in \mathcal{X}_{o}$,

$$
R_{01}(\cdot) \ll R_{01}^{t_{0} z}(\cdot):=R\left(\left(X_{0}, X_{1}\right) \in \cdot \mid X_{t_{0}}=z\right),
$$

then (1) and (2) are equivalent.

Proof. • Proof of $(2) \Rightarrow(1)$. It is contained in Example 1.7. Note that Hypothesis $(2.8)$ is not necessary.

- Proof of $(1) \Rightarrow(2)$. Since $P$ is Markov, Theorem 1.5 applied with $t=t_{0}$ leads to

$$
\frac{d P}{d R}=\alpha\left(X_{\left[0, t_{0}\right]}\right) \beta\left(X_{\left[t_{0}, 1\right]}\right) \quad R \text {-a.e. }
$$

with $\alpha$ and $\beta$ two measurable nonnegative functions. But, since $P$ belongs to the reciprocal family of $R$, following Theorem 2.13, its Radon-Nikodym derivative is

$$
\frac{d P}{d R}=h\left(X_{0}, X_{1}\right)
$$

for some measurable nonnegative function $h$ on $\mathcal{X}^{2}$. This implies that

$$
\alpha\left(X_{\left[0, t_{0}\right]}\right) \beta\left(X_{\left[t_{0}, 1\right]}\right)=h\left(X_{0}, X_{1}\right), \quad R \text {-a.e. }
$$

which in turns implies that the functions $\alpha$ and $\beta$ have the form

$$
\alpha\left(X_{\left[0, t_{0}\right]}\right)=a\left(X_{0}, X_{t_{0}}\right) \text { and } \beta\left(X_{\left[t_{0}, 1\right]}\right)=b\left(X_{t_{0}}, X_{1}\right), R \text {-a.e. }
$$

with $a$ and $b$ two measurable nonnegative functions on $\mathcal{X}^{2}$. It follows that

$$
a(x, z) b(z, y)=h(x, y) \quad \forall(x, z, y) \in \mathcal{N}^{c} \subset \mathcal{X}^{3},
$$

where the set $\mathcal{N} \subset \mathcal{X}^{3}$ is $R_{0, t_{0}, 1}$-negligible. Now, with the notation

$$
\mathcal{N}_{z}:=\{(x, y) ;(x, z, y) \in \mathcal{N}\} \subset \mathcal{X}^{2},
$$

we obtain

$$
0=R_{0, t_{0}, 1}(\mathcal{N})=\int_{\mathcal{X}} R_{01}^{t_{0} z}\left(\mathcal{N}_{z}\right) R_{t_{0}}(d z)
$$

which implies that $R_{01}^{t_{0} z}\left(\mathcal{N}_{z}\right)=0$ for $R_{t_{0}}$-a.e. $z \in \mathcal{X}_{0}$. Due to condition (2.8), one deduces that there exists $z_{o} \in \mathcal{X}_{o}$ such that $R_{01}\left(\mathcal{N}_{z_{o}}\right)=0$. Taking $f_{0}=a\left(\cdot, z_{o}\right)$ and $g_{1}=b\left(z_{o}, \cdot\right)$, we see that

$$
h(x, y)=f_{0}(x) g_{1}(y), \quad R_{01}(d x d y) \text {-a.e., }
$$

which proves that $d P / d R$ has the form expressed in (2.7). 
Remarks 2.15.

(a) Since $R$ is Markov, condition (2.8) is equivalent to

$$
\forall z \in \mathcal{X}_{o}, \quad R_{01}(\cdot) \ll R\left(X_{0} \in \cdot \mid X_{t_{0}}=z\right) \otimes R\left(X_{1} \in \cdot \mid X_{t_{0}}=z\right) .
$$

(b) Without any additional condition on $R$, both assertions of the above theorem fail to be equivalent. We provide a counter-example by constructing a measure $R$ which does not satisfy condition (2.8) and a Markov measure $P$ whose density with respect to $R$ is not of the form (2.7).

Let $R$ be the Markov measure with state space $\mathcal{X}=\{\mathrm{a}, \mathrm{b}\}$, initial law $R_{0}=\left(\delta_{\mathrm{a}}+\delta_{\mathrm{b}}\right) / 2$ and infinitesimal generator $\left(\begin{array}{cc}0 & 0 \\ \lambda & -\lambda\end{array}\right)$ for some $\lambda>0$. The support of $R$ is concentrated on two types of paths: the paths that are identically equal to $a$ or $b$, and the other ones that start from $b$ with one jump onto a after an exponential waiting time in $(0,1)$ with law $\mathcal{E}(\lambda)$. We see that $R$ does not satisfy $(2.8)$. Indeed, for all $t \in(0,1)$,

(a) $R_{01}^{t \mathrm{a}}(\mathrm{b}, \mathrm{b})=0$, but $R_{01}(\mathrm{~b}, \mathrm{~b})=\frac{e^{-\lambda}}{2}>0$. Thus, $R_{01} \nless R_{01}^{t a}$.

(b) $R_{01}^{t \mathrm{~b}}(\mathrm{a}, \mathrm{a})=0$, but $R_{01}(\mathrm{a}, \mathrm{a})=\frac{1}{2}>0$. Thus, $R_{01} \nless R_{01}^{t \mathrm{~b}}$.

Consider the Markov measure $P$ which gives half mass to the deterministic constant paths equal to a or b. It is dominated by $R$ with density:

$$
\frac{d P}{d R}= \begin{cases}1, & \text { if } X \equiv \mathrm{a} \\ e^{\lambda}, & \text { if } X \equiv \mathrm{b} \\ 0, & \text { if } X_{0} \neq X_{1}\end{cases}
$$

This density $d P / d R$ does not have the product form (2.7), since the system

$$
\left\{\begin{array}{l}
f(\mathrm{a}) g(\mathrm{a})=1 \\
f(\mathrm{~b}) g(\mathrm{~b})=e^{\lambda} \\
f(\mathrm{~b}) g(\mathrm{a})=0
\end{array}\right.
$$

admits no solution. Remark that the functions $\alpha$ and $\beta$ defined in (2.9) could be chosen as follows: $\alpha(X)=\beta(X)=1$ if $X \equiv \mathrm{a}, \alpha(X)=1$ if $X \equiv \mathrm{b}$, $\beta(X)=e^{\lambda}$ if $X \equiv \mathrm{b}$ and $\alpha(X)=\beta(X)=0$ otherwise.

\section{Reciprocal measures are solutions of entropy minimizing problems}

We conclude this survey paper going back to the problem that was originally addressed by Schrödinger in [45] and developed in [46]. It was the starting point of the theory of time-reversed Markov [30] and reciprocal diffusion processes. A modern formulation of Schrödinger's problem is stated below at $\left(\mathrm{S}_{\mathrm{dyn}}\right)$.

Motivated by a probabilistic solution of this problem, Bernstein [3] introduced the notion of reciprocal process. It is likely that Bernstein wasn't aware of the fact that $\left(\mathrm{S}_{\mathrm{dyn}}\right)$ 's solution is not only reciprocal, but also Markov as was clearly demonstrated four decades later by Jamison in [28]. 
The new ingredient of this section is the relative entropy, or Kullback-Leibler divergence, introduced in [32]. The relative entropy of a measure $p$ with respect to another measure $r$ on a measurable space $\mathcal{Y}$ is given by

$$
H(p \mid r):=\int_{\mathcal{Y}} \log \left(\frac{d p}{d r}\right) d p \in[0,+\infty]
$$

when $p$ is dominated by $r$, and $+\infty$ otherwise.

\subsection{Schrödinger's problem}

This problem is of a statistical physics nature.

\section{Dynamical and static formulations of Schrödinger's problem}

Let us sketch some results which are presented in detail in the review paper [34] (see also [1] and the references therein too). The modern dynamical formulation of Schrödinger's problem is as follows. Take a reference measure $R$ on $\Omega=D([0,1], \mathcal{X})$ and fix two probability measures $\mu_{0}, \mu_{1}$ on $\mathcal{X}$ (the marginal constraints). The aim is to minimize $P \mapsto H(P \mid R)$ where $P$ varies in the set of all path measures such that $P_{0}=\mu_{0}$ and $P_{1}=\mu_{1}$. A concise statement of Schrödinger's dynamical problem is

$$
H(P \mid R) \rightarrow \min ; \quad P \in \operatorname{Proba}(\Omega): P_{0}=\mu_{0}, P_{1}=\mu_{1} \quad\left(\mathrm{~S}_{\mathrm{dyn}}\right)
$$

Projecting via $\left(X_{0}, X_{1}\right)$ this variational problem onto the set $\mathcal{X}^{2}$ of endpoint configurations, one obtains the following associated static formulation: minimize $\pi \mapsto H\left(\pi \mid R_{01}\right)$, where $\pi$ is subject to vary in the set of all probability measures on $\mathcal{X}^{2}$ with prescribed marginals $\pi_{0}(d x):=\pi(d x \times \mathcal{X})=\mu_{0}$ and $\pi(d y):=$ $\pi(\mathcal{X} \times d y)=\mu_{1}$. A concise statement of Schrödinger's static problem is

$$
H\left(\pi \mid R_{01}\right) \rightarrow \min ; \quad \pi \in \operatorname{Proba}\left(\mathcal{X}^{2}\right): \pi_{0}=\mu_{0}, \pi_{1}=\mu_{1}
$$

Let us recall the uniqueness result [34, Prop. 2.3] which was proved by Föllmer [23] in the special case of a Brownian diffusion with drift.

Proposition 3.1. The dynamical and static Schrödinger problems each admit at most one solution $\widehat{P}$ and $\widehat{\pi}$. If $\widehat{P}$ denotes the solution of $\left(\mathrm{S}_{\mathrm{dyn}}\right)$, then $\widehat{\pi}=\widehat{P}_{01}$ is the solution of $(\mathrm{S})$. Conversely, if $\widehat{\pi}$ solves $(\mathrm{S})$, then the solution of $\left(\mathrm{S}_{\mathrm{dyn}}\right)$ is

$$
\widehat{P}(\cdot)=\int_{\mathcal{X}^{2}} R^{x y}(\cdot) \widehat{\pi}(d x d y) \in \Re_{a c}(R) .
$$

Sketch of the proof. As strictly convex minimization problems, $\left(\mathrm{S}_{\mathrm{dyn}}\right)$ and (S) admit at most one solution. Using the disintegration formula

$$
H(P \mid R)=H\left(P_{01} \mid R_{01}\right)+\int_{\mathcal{X}^{2}} H\left(P^{x y} \mid R^{x y}\right) P_{01}(d x d y),
$$


one obtains $H\left(P_{01} \mid R_{01}\right) \leq H(P \mid R)$ with equality (when $H(P \mid R)<+\infty$ ) if and only if $P^{x y}=R^{x y}$ for $P_{01}$-almost all $(x, y) \in \mathcal{X}^{2}$, which corresponds to $P \in \mathfrak{R}_{a c}(R)$. Thus, $\widehat{P}$ is the solution of $\left(\mathrm{S}_{\mathrm{dyn}}\right)$ if and only if it disintegrates as (3.1).

The solution of $\left(\mathrm{S}_{\mathrm{dyn}}\right)$ is Markov.

We present an existence (and uniqueness) result for $\left(\mathrm{S}_{\mathrm{dyn}}\right)$ and $(\mathrm{S})$ which is proved in [34].

Theorem 3.2. Let $R$ be a reference Markov measure with identical ${ }^{3}$ marginal laws at time 0 and 1 , denoted by $m$. Suppose that $R$ satisfies the following assumptions:

(i) there exists $0<t_{0}<1$ and a measurable set $\mathcal{X}_{o} \subset \mathcal{X}$ such that $R_{t_{0}}\left(\mathcal{X}_{o}\right)>0$ and

$$
R_{01} \ll R\left(\left(X_{0}, X_{1}\right) \in \cdot X_{t_{0}}=z\right), \quad \forall z \in \mathcal{X}_{o} .
$$

(ii) there exists a nonnegative measurable function $A$ on $\mathcal{X}$ such that

$$
R_{01}(d x d y) \geq e^{-A(x)-A(y)} m(d x) m(d y) .
$$

Suppose also that the constraints $\mu_{0}$ and $\mu_{1}$ satisfy

$$
H\left(\mu_{0} \mid m\right)+H\left(\mu_{1} \mid m\right)<+\infty \text { and } \int_{\mathcal{X}} A d \mu_{0}+\int_{\mathcal{X}} A d \mu_{1}<+\infty .
$$

Then (S) admits a unique solution $\widehat{\pi}$. It satisfies

$$
\widehat{\pi}(d x d y)=f_{0}(x) g_{1}(y) R_{01}(d x d y)
$$

for some $m$-measurable nonnegative functions $f_{0}, g_{1}: \mathcal{X} \rightarrow[0, \infty)$ which solve the so-called Schrödinger system:

$$
\left\{\begin{array}{lll}
f_{0}(x) E_{R}\left[g_{1}\left(X_{1}\right) \mid X_{0}=x\right] & =d \mu_{0} / d m(x), & \text { for } m \text {-a.e. } x \\
g_{1}(y) E_{R}\left[f_{0}\left(X_{0}\right) \mid X_{1}=y\right] & =d \mu_{1} / d m(y), & \text { for } m \text {-a.e. } y
\end{array}\right.
$$

Moreover, $\left(\mathrm{S}_{\mathrm{dyn}}\right)$ admits the unique solution

$$
\widehat{P}=f_{0}\left(X_{0}\right) g_{1}\left(X_{1}\right) R .
$$

It inherits the Markov property from $R$.

Remark 3.3. In the Schrödinger system, $E_{R}\left[f_{0}\left(X_{0}\right) \mid X_{1}\right]$ and $E_{R}\left[g_{1}\left(X_{1}\right) \mid X_{0}\right]$ are well defined even if $f_{0}\left(X_{0}\right)$ and $g_{1}\left(X_{1}\right)$ are not $R$-integrable. In fact, $f_{0}$ and $g_{1}$ are measurable and nonnegative; therefore, only positive integration is needed, see [33].

Generalizing Proposition 3.1, we obtain without additional effort the following result.

Corollary 3.4. Let $R$ be any reciprocal measure. The solution $\widehat{P}$ of the variational problem $\left(\mathrm{S}_{\mathrm{dyn}}\right)$, if it exists, belongs to the reciprocal family $\mathfrak{R}_{a c}(R)$.

\footnotetext{
${ }^{3}$ This restriction is done for simplifying the statements. We mostly have in mind a stationary reference measure $R$.
} 
A connection between Schrödinger's problem and PDEs

We give a PDE interpretation of the time-marginal flow $\left(\widehat{P}_{t}\right)_{0 \leq t \leq 1}$ of the solution $\widehat{P}$ of $\left(\mathrm{S}_{\mathrm{dyn}}\right)$, with the aim of clarifying its dynamical content. Let us come back to Example 1.12(i), where $\mathcal{X}=\mathbb{R}, m(d x)=d x, R$ denotes the (unbounded ${ }^{4}$ ) reversible Wiener measure and $r(s, x ; t, y)$ is its Gaussian kernel. Let us call $\rho_{0}(x)=\frac{d \mu_{0}}{d x}(x)$ and $\rho_{1}(y)=\frac{d \mu_{1}}{d y}(y)$. Then the system (3.2) reduces to

$$
\left\{\begin{array}{l}
f_{0}(x) \int r(0, x ; 1, y) g_{1}(y) d y=\rho_{0}(x) \\
g_{1}(y) \int f_{0}(x) r(0, x ; 1, y) d x=\rho_{1}(y) .
\end{array}\right.
$$

Schrödinger addressed the problem of the existence and uniqueness of solutions $\left(f_{0}, g_{1}\right)$ of this nonlinear system, given $r$ and the probabilistic boundary data $\rho_{0}$ and $\rho_{1}$. For $f_{0}$ and $g_{1}$ strictly positive, and $r$ considerably more general than the Gaussian kernel, introducing an entropy minimizing problem close to (S), Beurling [4] answered positively to this question. This was extended later by several authors, see [24, 34] for instance, taking advantage of the tight connection between (3.4) and $(\mathrm{S})$.

Let us denote by $f(t, z)$ the solution of the parabolic initial value problem

$$
\begin{cases}\left(-\partial_{t}+\partial_{z z}^{2} / 2\right) f=0, & 0<t \leq 1 \\ f(0, \cdot)=f_{0}, & t=0\end{cases}
$$

and by $g(t, z)$ the solution of the adjoint final value problem

$$
\begin{cases}\left(\partial_{t}+\partial_{z z}^{2} / 2\right) g=0, & 0 \leq t<1 \\ g(1, \cdot)=g_{1}, & t=1\end{cases}
$$

Remark that $f(t, z)=E_{R}\left(f_{0}\left(X_{0}\right) \mid X_{t}=z\right)$ and $g(t, z)=E_{R}\left(g_{1}\left(X_{1}\right) \mid X_{t}=z\right)$. Thanks to the Markov property of $R$, Theorem 1.2-(3) entails that for all $0 \leq$ $t \leq 1$

$$
\widehat{P}_{t}(d z)=f(t, z) g(t, z) d z .
$$

This relation is analogous to Born's formula:

$$
\rho_{t}(d z)=\psi_{t}(z) \overline{\psi_{t}(z)} d z
$$

where $\rho$ is the probability of presence of the quantum particle and $\psi$ is the wave function. Indeed, as remarked in 1928 by the astrophysicist Eddington (this is quoted in [46]), the relation between Schrödinger's equation and its complex conjugate can be interpreted as time reversal. Therefore, $\psi_{t}$ and $\bar{\psi}_{t}$ can be interpreted as two wave functions carrying respectively information from past and future. Indeed, they solve the standard quantum Schrödinger equation with respect to both directions of time. Switching to the classical statistical physics problem $\left(\mathrm{S}_{\mathrm{dyn}}\right)$, one sees that the functions $f_{t}$ and $g_{t}$ share similar properties,

\footnotetext{
${ }^{4}$ See [33] for the technical modifications that are necessary to handle the case of an unbounded reference measure.
} 
replacing the complex-valued Schrödinger equations in both directions of time by the heat equations (3.5) and (3.6). This striking analogy was Schrödinger's main motivation for introducing $\left(\mathrm{S}_{\mathrm{dyn}}\right)$. See [46] and also [10] and $[34, \S 6,7]$ for further detail.

Regarded as an element of $L^{2}(\mathbb{R}, d z)$ the solutions of (3.5) and (3.6) are analytic in the domain $\mathcal{R} e(t)>0$, continuous for $\mathcal{R} e(t) \geq 0$ and their values on the imaginary axis respectively solve the (quantum mechanical) Schrödinger equation and its complex conjugate. It is in this way that the Markov measure $\widehat{P}$ is a quantum-like measure. The multiplicative structure of the density $d \widehat{P}_{t} / d z$ appears as a stochastic analytic version of the complex conjugation of quantum functionals. When the Markov generator associated with $R$ is not self-adjoint, the same idea holds. For (much) more on this PDE connection, see [52]. This quantum mechanical connection is the starting point of a stochastic deformation of classical mechanics [54].

\subsection{A modification of Schrödinger's problem}

Having in mind these considerations about Schrödinger's problem, it appears that the notion of reciprocal measure was a technical intermediate step on the way to the solution of $\left(\mathrm{S}_{\mathrm{dyn}}\right)$. Indeed, Theorem 3.2 insures that $\widehat{P}$ is Markov, which is more specific than being reciprocal, and its proof doesn't rely on the reciprocal property. Nevertheless, there exist instances of non-Markov reciprocal measures that are interesting in their own right. Let us give a short presentation of two problems relating entropy minimization and reciprocal measures which are not Markov.

\section{Reciprocal measures and entropy minimization}

Consider the following modification of Schrödinger's problem

$$
H(P \mid R) \rightarrow \min ; \quad P \in \operatorname{Proba}(\Omega): P_{01}=\pi
$$

where $R$ is Markov and $\pi \in \operatorname{Proba}\left(\mathcal{X}^{2}\right)$ is given. Mimicking the sketch of proof of Proposition 3.1, it is easy to show that $\left(\mathrm{S}^{\pi}\right)$ admits a solution if and only if $H\left(\pi \mid R_{01}\right)<\infty$ and that, when this occurs, this solution is unique and is equal to

$$
R^{\pi}(\cdot):=\int_{\mathcal{X}^{2}} R^{x y}(\cdot) \pi(d x d y)
$$

When $\pi(d x d y)=f_{0}(x) g_{1}(y) R_{01}(d x d y)$ with $\left(f_{0}, g_{1}\right)$ solution of the Schrödinger system $(3.2)$, then $\left(\mathrm{S}^{\pi}\right)=\left(\mathrm{S}_{\mathrm{dyn}}\right)$. By $(2.5)$, we see that $R^{\pi}$ belongs to the reciprocal family $\mathfrak{R}_{a c}(R)$ of $R$. More precisely, when $\pi$ describes $\operatorname{Proba}\left(\mathcal{X}^{2}\right)$, defining

$$
\mathfrak{R}_{H}(R):=\left\{P: P \text { solution of }\left(\mathrm{S}^{\pi}\right) \text { with } \pi \in \operatorname{Proba}\left(\mathcal{X}^{2}\right)\right\},
$$

we see that

$$
\mathfrak{R}_{H}(R)=\left\{R^{\pi} ; \quad \pi \in \operatorname{Proba}\left(\mathcal{X}^{2}\right): H\left(\pi \mid R_{01}\right)<\infty\right\}
$$


which is a little smaller than $\mathfrak{R}_{a c}(R)$ for which $\pi$ is only required to satisfy $\pi \ll R_{01}$. Notice that

$$
\mathfrak{R}_{H}(R) \subset \mathfrak{R}_{a c}(R) \subset \mathfrak{R}(R)
$$

where these three classes are convex subsets of $\operatorname{Proba}(\Omega)$.

\section{Loop measures}

Example 2.4 exhibits a reciprocal loop measure $P_{\text {loop }}=\int_{\mathcal{X}} R^{x x} m(d x)$ which is not Markov in general. Denoting $\pi_{m}(d x d y)=m(d x) \delta_{x}(d y)$, we see that $P_{\text {loop }}=R^{\pi_{m}}$.

Remark that in the important case where $R$ is the reversible Brownian motion $^{5}$, then $\pi_{m} \nless R_{01}$ because $R_{01}\left(X_{0}=X_{1}\right)=0$ and $\pi_{m}\left(X_{0}=X_{1}\right)=1$. Consequently, ( $\left.\mathrm{S}^{\pi_{m}}\right)$ has no solution. The endpoint constraint $\pi=\pi_{m}$ of $\left(\mathrm{S}^{\pi}\right)$ is degenerate in the same way as $\left(\mu_{0}, \mu_{1}\right)=\left(\delta_{x}, \delta_{y}\right)$ is a degenerate constraint of $\left(\mathrm{S}_{\mathrm{dyn}}\right)$. Indeed, both $\pi_{m}$ and $\delta_{(x, y)}$ verify $H\left(\pi_{m} \mid R_{01}\right), H\left(\delta_{(x, y)} \mid R_{01}\right)<\infty$ and can be approximated by finite entropy constraints.

Stochastic representation of incompressible hydrodynamical flows

Consider the following entropy minimization problem

$$
H(P \mid R) \rightarrow \min ; \quad P \in \operatorname{Proba}(\Omega): P_{t}=m, \forall 0 \leq t \leq 1, P_{01}=\pi
$$

which consists of minimizing the relative entropy $H(P \mid R)$ of the path measure $P$ with respect to the Markov measure $R$ subject to the constraints that the time marginal flow $\left(P_{t}\right)_{0 \leq t \leq 1}$ is constantly equal to a given $m \in \operatorname{Proba}(\mathcal{X})$ and that the endpoint marginal $P_{01}$ is equal to a given $\pi \in \operatorname{Proba}\left(\mathcal{X}^{2}\right)$. This problem is a natural stochastization of Arnold's approach to the Euler equation for incompressible fluids [2] which is connected to the Navier-Stokes equation. The justification of this assertion is part of a work in progress by two of the authors. The incompressibility constraints is $P_{t}=m, \forall 0 \leq t \leq 1$ when $m$ is the volume measure on the manifold $\mathcal{X}$. The constraint $P_{01}=\pi$ is Brenier's relaxation [5] of Arnold's final diffeomorphism. It can be proved using the results of the present paper that for a generic endpoint constraint $\pi$, the minimizer of (3.7) (whenever it exists) is reciprocal but not Markov.

\section{Acknowledgements}

The authors thank the referee for useful comments and especially for indicating the reference [18].

\footnotetext{
${ }^{5}$ See [33] for the technical modifications that are necessary to handle the case of an unbounded reference measure.
} 


\section{References}

[1] AebI, R. (1996). Schrödinger Diffusion Processes. Birkhäuser. MR1391719

[2] Arnold, V. (1966). Sur la géométrie différentielle des groupes de Lie de dimension infinie et ses applications à l'hydrodynamique des fluides parfaits. Ann. Inst. Fourier 16, 1, 319-361. MR0202082

[3] Bernstein, S. (1932). Sur les liaisons entre les grandeurs aléatoires. Verhand. Internat. Math. Kongr. Zürich, Band I.

[4] Beurling, A. (1960). An automorphism of product measures. Ann. of Math. 72, 189-200. MR0125424

[5] Brenier, Y. (1989). The least action principle and the related concept of generalized flows for incompressible perfect fluids. J. Amer. Math. Soc. 2, 2, 225-255. MR0969419

[6] Chaumont, L. and Uribe-Bravo, G. (2011). Markovian bridges: Weak continuity and pathwise constructions. Ann. Probab. 39, 609-647. MR2789508

[7] Chay, S. (1972). On quasi-Markov random fields. J. Multivariate Anal. 2, 14-76. MR0303589

[8] Chung, K. (1968). A Course in Probability Theory. Harcourt, Brace \& World, Inc., New York. MR0229268

[9] Chung, K. And Walsh, J. (2005). Markov Processes, Brownian Motion and Time Symmetry. Lecture Notes in Mathematics. Springer Verlag, New York. MR2152573

[10] Chung, K. and Zambrini, J.-C. (2008). Introduction to Random Time and Quantum Randomness. World Scientific. MR1999000

[11] Clark, J. (1991). A local characterization of reciprocal diffusions. Applied Stoch. Analysis 5, 45-59. MR1108416

[12] Conforti, G., Dai Pra, P., And Relly, S., Reciprocal classes of jump processes. Preprint. http://opus.kobv.de/ubp/volltexte/2014/7077/ pdf/premath06.pdf.

[13] Conforti, G. And LÉOnard, C., Reciprocal classes of random walks on graphs. In preparation.

[14] Conforti, G., Léonard, C., Murr, R., and Relly, S., Bridges of Markov counting processes. Reciprocal classes and duality formulas. Preprint, arXiv:1408.1332.

[15] Courrège, P. and Renouard, P. (1975). Oscillateurs harmoniques mesures quasi-invariantes sur $c(\mathbb{R}, \mathbb{R})$ et théorie quantique des champs en dimension 1. In Astérisque. Vol. 22-23. Société Mathématique de France. MR0496165

[16] Cruzeiro, A., Wu, L., And Zambrini, J.-C. (2000). Bernstein processes associated with a Markov process. In Stochastic Analysis and Mathematical Physics, ANESTOC'98. Proceedings of the Third International Workshop, R. Rebolledo, Ed., Trends in Mathematics. Birkhäuser, Boston, 41-71. MR1764785

[17] Cruzeiro, A. And Zambrini, J.-C. (1991). Malliavin calculus and Euclidean quantum mechanics, I. J. Funct. Anal. 96, 1, 62-95. MR1093507 
[18] Dang Ngoc, N. And Yor, M. (1978). Champs markoviens et mesures de Gibbs sur $\mathbb{R}$. Ann. Sci. Éc. Norm. Supér., 29-69. MR0504421

[19] Doob, J. (1953). Stochastic Processes. Wiley. MR0058896

[20] Doob, J. (1957). Conditional Brownian motion and the boundary limits of harmonic functions. Bull. Soc. Math. Fr. 85, 431-458. MR0109961

[21] Dynkin, E. (1961). Theory of Markov Processes. Prentice-Hall Inc.

[22] Fitzsimmons, P., Pitman, J., And Yor, M. (1992). Markovian bridges: Construction, Palm interpretation, and splicing. Progr. Probab. 33, 101134. MR1278079

[23] Föllmer, H. (1988). Random fields and diffusion processes, in École d'été de Probabilités de Saint-Flour XV-XVII-1985-8\%. Lecture Notes in Mathematics, Vol. 1362. Springer, Berlin. MR0983373

[24] Föllmer, H. AND Gantert, N. (1997). Entropy minimization and Schrödinger processes in infinite dimensions. Ann. Probab. 25, 2, 901-926. MR1434130

[25] Georgit, H.-O. (2011). Gibbs measures and phase transitions. In Studies in Mathematics, Second ed. Vol. 9. Walter de Gruyter. MR2807681

[26] Jamison, B. (1970). Reciprocal processes: The stationary Gaussian case. Ann. Math. Statist. 41, 1624-1630. MR0267637

[27] Jamison, B. (1974). Reciprocal processes. Z. Wahrsch. verw. Geb. 30, 65-86. MR0359016

[28] Jamison, B. (1975). The Markov processes of Schrödinger. Z. Wahrsch. verw. Geb. 32, 4, 323-331. MR0383555

[29] KallenberG, O. (1981). Splitting at backward times in regenerative sets. Annals Probab. 9, 781-799. MR0628873

[30] Kolmogorov, A. (1936). Zur Theorie der Markoffschen Ketten. Mathematische Annalen 112.

[31] Krener, A. (1988). Reciprocal diffusions and stochastic differential equations of second order. Stochastics 24, 393-422. MR0972972

[32] Kullback, S. And Leibler, R. (1951). On information and sufficiency. Annals of Mathematical Statistics 22, 79-86. MR0039968

[33] LÉOnARD, C., Some properties of path measures. To appear in Séminaire de probabilités de Strasbourg, vol. 46. Preprint, arXiv:1308.0217.

[34] LÉOnARD, C. (2014). A survey of the Schrödinger problem and some of its connections with optimal transport. Discrete Contin. Dyn. Syst. A 34, 4, 1533-1574. MR3121631

[35] Meyer, P.-A. (1967). Processus de Markov. Lecture Notes in Mathematics, Vol. 26. Springer Verlag, New York. MR0219136

[36] MurR, R. (2012). Reciprocal classes of Markov processes. An approach with duality formulae. Ph.D. thesis, Universität Potsdam, http://opus. kobv.de/ubp/volltexte/2012/6301.

[37] Nagasawa, M. (1993). Schrödinger Equations and Diffusions Theory. Monographs in Mathematics, Vol. 86. Birkhäuser. MR1227100

[38] Nelson, E. (1967). Dynamical Theories of Brownian Motion. Princeton University Press. Second edition (2001) at: http://www.math.princeton. $\mathrm{edu} / \sim$ nelson/books.html. MR0214150 
[39] Osada, H. And Spohn, H. (1999). Gibbs measures relative to Brownian motion. Annals Probab. 27, 1183-1207. MR1733145

[40] Privault, N. And Zambrini, J.-C. (2004). Markovian bridges and reversible diffusions with jumps. Ann. Inst. H. Poincaré. Probab. Statist. 40, 599-633. MR2086016

[41] ReLLy, S. (2014). Reciprocal processes. A stochastic analysis approach. In Modern Stochastics with Applications. Optimization and Its Applications, Vol. 90. Springer, 53-67.

[42] Relly, S. and Thieullen, M. (2004). A characterization of reciprocal processes via an integration by parts formula on the path space. Probab. Theory Related Fields 123, 97-120. MR1906440

[43] Rellly, S. And Thieullen, M. (2005). Duality formula for the bridges of a brownian diffusion: Application to gradient drifts. Stochastic Processes and Their Applications 115, 1677-1700. MR2165339

[44] Royer, G. ANd Yor, M. (1976). Représentation intégrale de certaines mesures quasi-invariantes sur $c(\mathbb{R})$; mesures extrémales et propriété de Markov. Ann. Inst. Fourier 26, 7-24. MR0447517

[45] Schrödinger, E. (1931). Über die Umkehrung der Naturgesetze. Sitzungsberichte Preuss. Akad. Wiss. Berlin. Phys. Math. 144, 144-153.

[46] Schrödinger, E. (1932). Sur la théorie relativiste de l'électron et l'interprétation de la mécanique quantique. Ann. Inst. H. Poincaré 2, 269310. MR1508000

[47] Thieullen, M. (1993). Second order stochastic differential equations and non-gaussian reciprocal diffusions. Probab. Theory Related Fields 97, 231257. MR1240725

[48] Thieullen, M. (2002). Reciprocal diffusions and symmetries of parabolic PDE: The nonflat case. Potential Analysis 16, 1, 1-28. MR1880345

[49] Thieullen, M. and Zambrini, J.-C. (1997a). Probability and quantum symmetries. I. The theorem of Noether in Schrödinger's Euclidean quantum mechanics. Ann. Inst. H. Poincaré Phys. Théor. 67, 297-338. MR1472821

[50] Thieullen, M. And Zambrini, J.-C. (1997b). Symmetries in the stochastic calculus of variations. Probab. Theory Related Fields 107, 3, 401-427. MR1440139

[51] van Putten, C. And van Schuppen, J. (1985). Invariance properties of the conditional independence relation. Ann. Probab. 13, 934-945. MR0799429

[52] Vuillermot, P. And Zambrini, J.-C. (2012). Bernstein diffusions for a class of linear parabolic PDEs. Journal of Theor. Probab., 1-44.

[53] Wentzell, A. (1981). A course in the theory of stochastic processes. Mc Graw-Hill.

[54] Zambrini, J.-C., The research program of stochastic deformation (with a view toward geometric mechanics). To be published in Stochastic Analysis. A Series of Lectures. Birkhaüser. Preprint, arXiv:1212.4186.

[55] Zambrini, J.-C. (1986). Variational processes and stochastic version of mechanics. Journal of Mathematical Physics 27, 2307-2330. MR0854761 